\title{
The ISO-LWS map of the Serpens cloud core*
}

\section{The line spectra}

\author{
B. Larsson ${ }^{1}$, R. Liseau ${ }^{1}$, and A. B. Men'shchikov ${ }^{1,2}$ \\ 1 Stockholm Observatory, SCFAB, Roslagstullsbacken 21, 10691 Stockholm, Sweden \\ e-mail: bem@astro.su.se; rene@astro.su.se \\ 2 Max-Planck-Institut für Radioastronomie, Auf dem Hügel, Bonn, Germany \\ e-mail: sasha@mpifr-bonn.mpg.de
}

Received 30 November 2001 / Accepted 29 January 2002

\begin{abstract}
We present spectrophotometric ISO imaging with the LWS and the CAM-CVF of the Serpens molecular cloud core. The LWS map is centred on the far infrared and submillimetre source FIRS 1/SMM 1 and its size is $8^{\prime} \times 8^{\prime}$. The fine structure line emission in [O I] $63 \mu \mathrm{m}$ and $[\mathrm{C} \mathrm{II}] 157 \mu \mathrm{m}$ is extended on the arcminute scale and can be successfully modelled to originate in a PDR with $G_{0}=15 \pm 10$ and $n\left(\mathrm{H}_{2}\right)$ in the range of $\left(10^{4}-10^{5}\right) \mathrm{cm}^{-3}$. Extended emission might also be observed in the rotational line emission of $\mathrm{H}_{2} \mathrm{O}$ and high- $J$ CO. However, lack of sufficient angular resolution prevents us from excluding the possibility that the emssion regions of these lines are point like, which could be linked to the embedded objects SMM 9/S 68 and SMM 4. Toward the Class 0 source SMM 1, the LWS observations reveal, in addition to fine structure line emission, a rich spectrum of molecular lines, superposed onto a strong, optically thick dust continuum (Larsson et al. 2000). The sub-thermally excited and optically thick $\mathrm{CO}, \mathrm{H}_{2} \mathrm{O}$ and $\mathrm{OH}$ lines are tracing an about $10^{3} \mathrm{AU}$ source with temperatures higher than $300 \mathrm{~K}$ and densities above $10^{6} \mathrm{~cm}^{-3}\left(M=0.01 M_{\odot}\right)$. The molecular abundances, $X=N(\mathrm{~mol}) / N\left(\mathrm{H}_{2}\right)$, are $X=$ $(1,0.1,0.02, \geq 0.025) \times 10^{-4}$ for $\mathrm{CO}, \mathrm{H}_{2} \mathrm{O}, \mathrm{OH}$ and ${ }^{13} \mathrm{CO}$, respectively. Our data are consistent with an ortho-topara ratio of 3 for $\mathrm{H}_{2} \mathrm{O}$. OH appears highly overabundant, which we tentatively ascribe to an enhanced (X-ray) ionisation rate in the Serpens cloud core $\left(\zeta \gg 10^{-18} \mathrm{~s}^{-1}\right)$. We show that geometry is of concern for the correct interpretation of the data and based on 2D-radiative transfer modelling of the disk/torus around SMM 1, which successfully reproduces the entire observed SED and the observed line profiles of low-to-mid- $J$ CO isotopomers, we can exclude the disk to be the source of the LWS-molecular line emission. The same conclusion applies to models of dynamical collapse ("inside-out" infall). The 6 " pixel resolution of the CAM-CVF permits us to see that the region of rotational $\mathrm{H}_{2}$ emission is offset from SMM 1 by $30^{\prime \prime}$, at position angle $340^{\circ}$, which is along the known jet flow from the Class 0 object. This $\mathrm{H}_{2}$ gas is extinguished by $A_{\mathrm{V}}=4.5$ mag and at a temperature of $10^{3} \mathrm{~K}$, which suggests that the heating of the gas is achieved through relatively slow shocks. Although we are not able to establish any firm conclusion regarding the detailed nature of the shock waves, our observations of the molecular line emission from SMM 1 are to a limited extent explainable in terms of an admixture of $J$-shocks and of $C$-shocks, the latter with speeds of about $(15-20) \mathrm{km} \mathrm{s}^{-1}$, whereas dynamical infall is not directly revealed by our data.
\end{abstract}

Key words. ISM: individual objects: Serpens cloud core, FIRS 1/SMM 1 - ISM: abundances - ISM: molecules ISM: clouds - ISM: jets and outflows - stars: formation

\section{Introduction}

The Serpens dark cloud is currently forming a dense cluster of low to intermediate mass stars (Strom et al. 1976; Kaas 1999). This star forming complex is situated in the inner Galaxy, not very far from the direction toward the Galactic Centre $\left(\ell^{\mathrm{II}}=32^{\circ}\right)$. At the distance of

Send offprint requests to: B. Larsson, e-mail: bem@astro.su.se

* Based on observations with ISO, an ESA project with instruments funded by ESA Member States (especially the PI countries: France, Germany, The Netherlands and the UK) and with the participation of ISAS and NASA.
$310 \mathrm{pc}$ (de Lara et al. 1991), the Serpens cloud core is only $30 \mathrm{pc}$ from the nominal galactic plane, i.e. well within the scale height of the molecular gas of about $80 \mathrm{pc}$ (Dame et al. 1987). Not totally unexpected, IRAS-observations revealed intense and patchy far infrared (FIR) emission on a sloping background. Earlier observations had discovered discrete FIR sources of relatively low luminosity (Nordh et al. 1982; Harvey et al. 1984).

The empirical classification scheme developed by Lada \& Wilking (1984), and later extended by André et al. (1993), is based on the Spectral Energy Distribution (SED) of the young stellar objects. In a previous paper 
(Larsson et al. 2000), we were discussing the SEDs of the submm-sources in the Serpens cloud core and for the dominating source, SMM1 (also known as Serpens FIRS 1; Harvey et al. 1984), we reached the conclusion that its SED classifies it as Class 0. The existence of a circumstellar disk has been announced by Brown et al. (2000). An immediate question is then, whether SMM1 shows any detectable or deducable spectroscopic evidence of disk accretion and/or of dynamical infall. Over a $0.2 \mathrm{pc}$ (about $2^{\prime}$ ) region toward the Serpens cloud core, Williams $\&$ Myers (2000) reported signs of infall. Observations toward SMM 1 of molecular line profiles (Mardones et al. 1997 and Gregersen et al. 1997), have not so far been able to reveal a clear "collapse signature". This is possibly because of confusion with the known mass outflow activity of the source (Rodríguez et al. 1989; Eiroa et al. 1992; McMullin et al. 1994; White et al. 1995; Davis et al. 1999; Williams \& Myers 2000; Testi et al. 2000) and/or different velocity components in the cloud complex. On the other hand, Hogerheijde et al. (1999) found, from continuum interferometric observations, the density profile of the source to be consistent with the theoretical expectation of a collapsing cloud.

The earlier molecular line results were based on observations of low excitation transitions, which are not particularly (or not all) sensitive to the conditions expected to prevail in the deeper layers of the source. In this paper, we present spectral line data of the Serpens cloud core, both for a spatial map and for pointed deep integrations, which contain lines also of very high excitation. These are potentially better suited to "penetrate" to regions which were previously hidden from view. Our interpretation of the results will especially focus on the evolution of this star forming complex.

In Sect. 2, we reiterate the LWS observations and a summary is given for the data reductions, whereas a more detailed account is provided in Appendix A. The resulting line spectra are presented in Sect. 3. These results are discussed at some depth in Sect. 4. We first exploit relatively simple analytical methods, which result in spatially averaged properties. These should be useful to limit the parameter space for more sophisticated numerical modelling. This is done for the transfer of both continuum and line radiation, and these models lead to some valuable conclusions. In a summarising discussion, we make an attempt to bring these various pieces of information into a coherent physical picture. Finally in Sect. 5, we summarise our main conclusions from this work.

\section{Observations and data reductions}

\subsection{The ISO observations}

A $5 \times 5$ spectrophotometric map in the far-infrared (FIR) of the Serpens cloud core was obtained with the LongWavelength Spectrometer (LWS; 43-197 $\mu \mathrm{m}, R_{\lambda}=140-$ 330) on board the Infrared Space Observatory (ISO) on October 21, 1996. The ISO-project is described by
Kessler et al. (1996) and the LWS is described by Clegg et al. (1996) and Swinyard et al. (1996).

The formal map centre, viz. $\alpha=18^{\mathrm{h}} 29^{\mathrm{m}} 50.29$ and $\delta=$ $1^{\circ} 15^{\prime} 18^{\prime \prime}$, epoch J 2000 , coincides to within $10^{\prime \prime}$ with the position of the sub-millimetre source SMM 1. The pointing accuracy in the map is determined as $1^{\prime \prime}(\mathrm{rms})$. The spacings between positions in the map, oriented along the equatorial coordinates, are $100^{\prime \prime}$. The size of the LWS-map is $8^{\prime} \times 8^{\prime}$, corresponding to $(0.7 \times 0.7=0.5) D_{310}^{2} \mathrm{pc}^{2}$, where $D_{310}$ denotes the distance to the Serpens cloud core in units of $310 \mathrm{pc}$.

At each map-point the grating of the LWS was scanned 6 times in fast mode, oversampling the spectral resolution at twice the Nyquist rate. Each position was observed for nearly $15 \mathrm{~min}$. The centre position was re-observed half a year later on April 15, 1997, for a considerably longer integration time (24 spectral scans).

In addition, imaging spectrophotometric data obtained with the Continuous Variable Filter (CVF; $5-16.5 \mu \mathrm{m}, 32 \times 32 \mathrm{pxl}$, pixel-FOV $\left.=6^{\prime \prime}, R_{\lambda} \gtrsim 35\right)$ of ISOCAM (Cesarsky et al. 1996) were also analysed. The detailed observing log is given in our previous paper (Larsson et al. 2000).

\subsection{The reduction of the ISO data}

\subsection{LWS data}

The LWS data reductions were done with the interactive analysis package LIA. This pipeline processing was done in two ways. For the long wave detectors LW 1LW 5, the Relative Spectral Response Functions (RSRFs) of the most recent version (OLP 10) provided an overall better agreement than the older OLP 8 for the detector inter-calibration. However, the OLP 10 RSRFs also contained a number of strong features, which resulted in spurious "lines" in the reduced spectra (Appendix A). We decided therefore to use the RSRFs of OLP 8, which did not show this behaviour, but scaled to the OLP 10 absolute levels. For the short-wave detectors SW 1-SW 5, no such difference between OLP 8 and OLP 10 was apparent and consequently we used the latest version. A detailed account for the first steps in the LWS reduction is given in Appendix A.

Subsequent post-pipeline processing used the package ISAP. At each position, the individual LWS scans for each of the 10 detectors were examined, "deglitched" and averaged. Corrections were applied to the "fringed" spectra in the map. The fringing indicates that the emission is extended and/or that point sources were not on the optical axis of the LWS, i.e. the radial distance from the optical axis was typically larger than about $25^{\prime \prime}$.

The absolute flux calibration is better than about $30 \%$ (Swinyard et al. 1996). Overlapping spectral regions of adjacent detectors were generally within 10\% ("detector stitching" uncertainty). At $60 \mu \mathrm{m}$ and $100 \mu \mathrm{m}$, the correspondance with broad-band IRAS data is even better than 10\% (Larsson et al. 2000). 

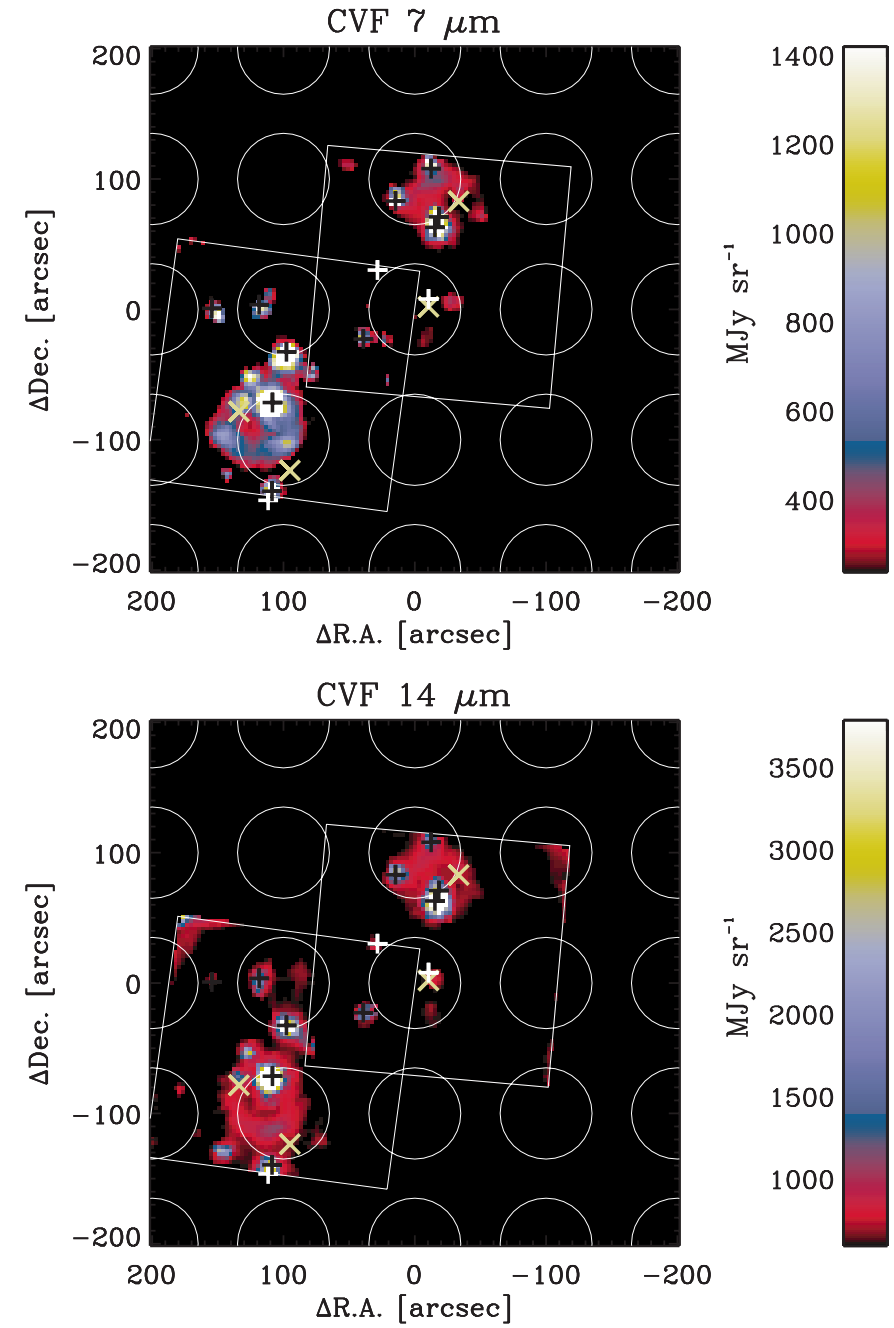

Fig. 1. The from CVF frames synthesised images at $7 \mu \mathrm{m}$ and $14 \mu \mathrm{m}$. From comparison with CAM broad-band observations at these wavelengths and deep K-images the point sources were identified (see: Kaas 1999). These are shown by plus-signs (white and black) and were used to align the CVF frames with the equatorial coordinate system in the sky. Major SMM sources in the field are shown by yellow crosses. The LWS map pointings are indicated by the contours of the FWHM of the beam (white circles).

\subsection{ISO-CAM-CVF data}

The ISO-CAM data were reduced with the CIA programs using OLP 8.4. This included dark current correction, transient correction and deglitching and flat field corrections. The calibrations were based on OLP 5.4. The proper alignment of the CVF fields was achieved with the aid of point sources identified in both CVF and broadband ( $7 \mu \mathrm{m}$ and $14 \mu \mathrm{m})$ CAM observations (Fig. 1).

\section{Results}

\subsection{Spectral imaging in $\mathrm{H}_{2}$}

Lines from purely rotationally excited $\mathrm{H}_{2}$ are clearly discernible in the CVF-image. In particular, all lines which fall in the observed spectral range, were detected and, in
Table 1. Observed $\mathrm{H}_{2}$ fluxes near SMM 1.

\begin{tabular}{lll}
\hline \hline Transition & \multicolumn{1}{c}{$\begin{array}{l}\text { Peak }^{\dagger} \\
\left(10^{-21} \mathrm{~W} \mathrm{~cm}^{-2}\right)\end{array}$} & $\begin{array}{l}\text { Total }^{\ddagger} \\
\left(10^{-20} \mathrm{~W} \mathrm{~cm}^{-2}\right)\end{array}$ \\
\hline $0-0 \mathrm{~S}(2)$ & $2.9 \pm 0.9$ & $1.5 \pm 0.2$ \\
$0-0 \mathrm{~S}(3)$ & $5.2 \pm 0.6$ & $4.8 \pm 0.4$ \\
$0-0 \mathrm{~S}(4)$ & $8.8 \pm 1.0$ & $3.6 \pm 0.3$ \\
$0-0 \mathrm{~S}(5)$ & $19.8 \pm 1.2$ & $7.0 \pm 0.5$ \\
$0-0 \mathrm{~S}(6)$ & $6.2 \pm 0.8$ & $0.7 \pm 0.2$ \\
$0-0 \mathrm{~S}(7)$ & $15.6 \pm 2.8$ & $5.8 \pm 0.6$ \\
$1-0 \mathrm{~S}(1)^{\amalg}$ & 2.5 & 1.0 \\
\hline
\end{tabular}

Notes to the table:

$\dagger$ Peak emission into a $36 \operatorname{arcsec}^{2}$ pixel.

$¥$ Total emission over a source of $350 \operatorname{arcsec}^{2}$.

$\amalg$ Estimated from Fig. 3 of Eiroa \& Casali (1989).

Table 1, their observed fluxes are listed. These refer to the 3 para- $\mathrm{H}_{2}$ lines $0-0 \mathrm{~S}(2) 12.3, \mathrm{~S}(4) 8.0$ and $\mathrm{S}(6) 6.1 \mu \mathrm{m}$ and the 3 ortho- $\mathrm{H}_{2}$ lines $0-0 \mathrm{~S}(3) 9.7, \mathrm{~S}(5) 6.9$ and $\mathrm{S}(7) 5.5 \mu \mathrm{m}$. Figure 2 shows maps near SMM 1 in these six lines, delineating the spatial distribution of the rotationally excited $\mathrm{H}_{2}$ gas along the known jet-flow from SMM 1 (Eiroa \& Casali 1989; Hodapp 1999). In addition, somewhat weaker $\mathrm{H}_{2}$ emission is also observed near SMM 3, the discussion of which will be postponed to a future paper.

\subsection{LWS-maps of [O I] $63 \mu \mathrm{m},[\mathrm{C} ॥] 157 \mu \mathrm{m}$, ortho- $\mathrm{H}_{2} \mathrm{O} 179.5 \mu \mathrm{m}$ and $\mathrm{CO} 186 \mu \mathrm{m}$}

Within the $8^{\prime} \times 8^{\prime}$ map observed with the LWS, the spatial distribution of the fine structure lines [O I] $63 \mu \mathrm{m}$ and [C II] $157 \mu \mathrm{m}$ is shown in Fig. 3 together with that of the rotational lines of $\mathrm{H}_{2} \mathrm{O}\left(2_{12}-1_{01}\right)$ (connecting to the ground state) and $\mathrm{CO}(J=14-13)$ (lowest $J$-transition admitted and close in wavelength to the $\mathrm{H}_{2} \mathrm{O}$ line).

Like the emission in [C II] $157 \mu \mathrm{m}$, [O I] $63 \mu \mathrm{m}$ emission is observed in each position of our map, but with maximum intensity at the position of SMM 1. Secondary maxima are also seen along a ridge toward SMM $4 / 3 / 8$ in the southeast-to-east. In addition, the emission is extended toward SMM 9/S68 NW. The overall [O I] $63 \mu \mathrm{m}$ distribution is strikingly similar to that seen in the mm-regime in a variety of high density tracing molecules (McMullin et al. 2000). Very interesting is also the fact that, just in the northwest corner of the map, the shocked flow HH 460 (Davis et al. 1999; Ziener \& Eislöffel 1999) seems to be discernible.

In [C II] $157 \mu \mathrm{m}$, maximum emission is seen toward S 68 (Sharpless 1959) and the cluster in the southeast, whereas SMM 1 is inconspicuous in this map. To some extent, the $[\mathrm{C}$ II $] 157 \mu \mathrm{m}-\mathrm{map}$ is an inverted image of that seen in [O I] $63 \mu \mathrm{m}$.

The molecular emision in the $179.5 \mu \mathrm{m}$ line of ortho$\mathrm{H}_{2} \mathrm{O}$ and in the $186 \mu \mathrm{m}$ line of $\mathrm{CO}$ is strongest toward SMM 1, but displays also some extensions along the 

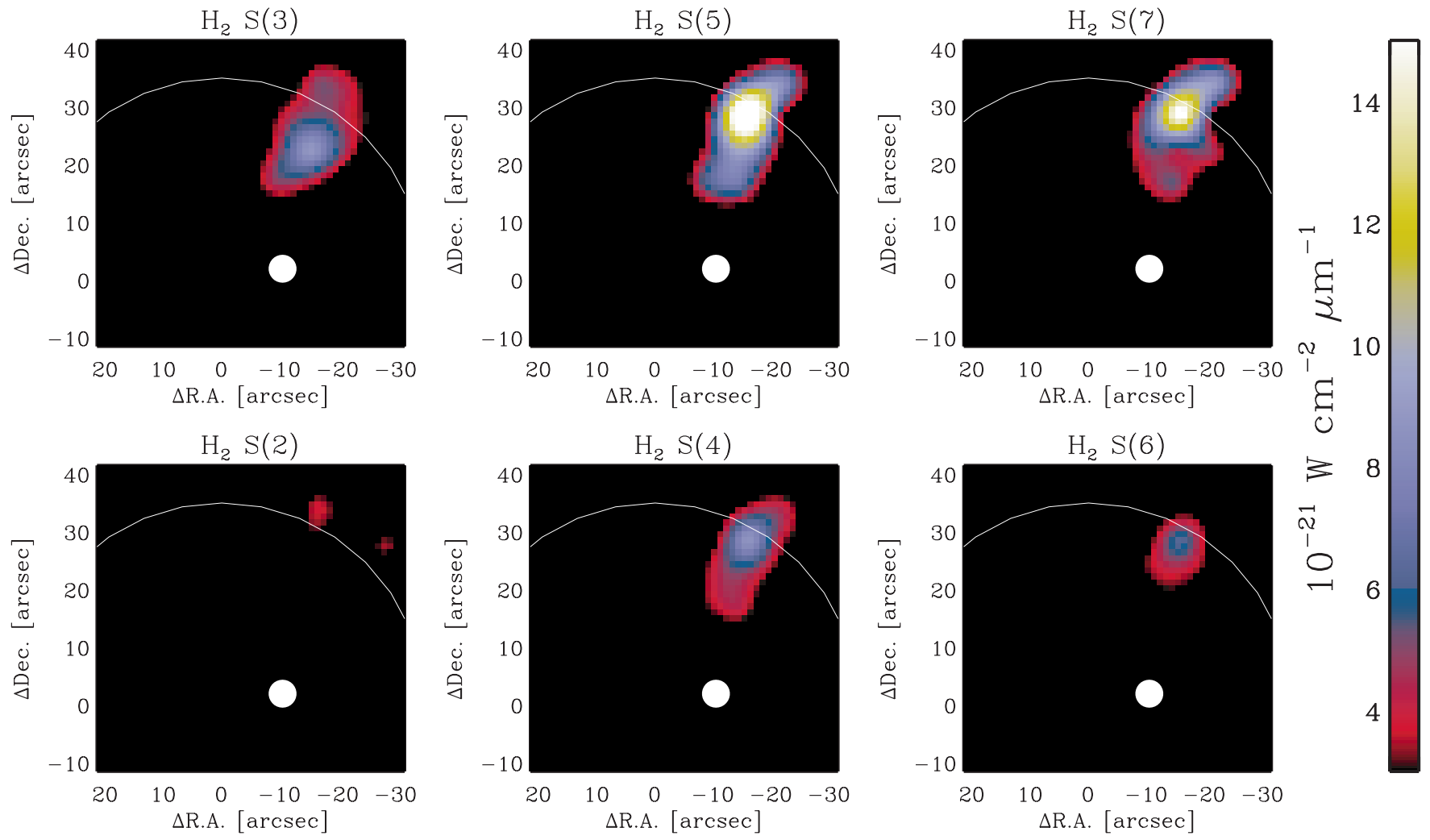

Fig. 2. CVF images toward SMM 1 in 3 ortho- $\mathrm{H}_{2}$ (upper frames) and 3 para- $\mathrm{H}_{2}$ (lower row) lines. Offsets are in arcsec and relative to the centre position $(0,0)$ of the LWS map. The white arcs outline the FWHM contour of the LWS beam $\left(70^{\prime \prime}\right)$ and the white spot designates the position of SMM 1 . The actual CVF pixels are square $6^{\prime \prime}$.

[O I] $63 \mu \mathrm{m}$ ridge, i.e. toward the northwest and the southeast. The spatial resolution is not sufficient, however, to decide whether this emission is extended or due to the numerous SMM-sources in the region (cf. Fig. 3).

\subsection{The line spectrum of SMM 1 from 43 to $197 \mu m$}

The continuum subtracted line spectrum of SMM 1 from 43 to $197 \mu \mathrm{m}$ is displayed in Fig. 4, revealing a plethora of emission lines from both atomic and molecular species. In the figure, also the wavelength coverage of the ten individual LWS detectors is indicated (cf. Sect. 2.2). The identification of the lines and their parameters, as obtained from profile fitting, are listed in Tables 2 to 5. Listed are the identified species, the upper and lower states and the rest wavelength of the transition. This is followed by the observed wavelength with the measurement error and the difference between the observed and the rest wavelength. Then the line width and the line flux with fittingerrors, respectively, are tabulated and the error estimate for the line flux, i.e. $\Delta F=\sqrt{\sigma_{\mathrm{l}}^{2}+\sigma_{\mathrm{c}}^{2}}$ where $\sigma_{\mathrm{l}}$ is the fitting error and $\sigma_{\mathrm{c}}$ is the rms-level of the surrounding continuum integrated over one spectral resolution element. In the last two columns, the type of the Gauss fitting (single or multiple component) and the LWS detector are given.

Out of a total of 8 measured CO lines, clear detections are found for 7 transitions. In addition, we identfy 5 (possibly 8) lines of ortho- $\mathrm{H}_{2} \mathrm{O}$, and 1 (possibly 2) of para- $\mathrm{H}_{2} \mathrm{O}$. Further, 4 lines of $\mathrm{OH}$ are clearly present in the spectrum, whereas for two more lines this status is less clear. Aside from the line emission from molecules, lines from $\mathrm{O}^{0}$ and $\mathrm{C}^{+}$are also present. Possible implications from these results will be discussed in the following sections.

\section{Discussion}

\subsection{The atomic line spectrum: CNO}

\subsection{1. [O III] $53 \mu \mathrm{m},[\mathrm{O} I I]) 88 \mu \mathrm{m}$ and [N II] $122 \mu \mathrm{m}$}

As is evident from Table 2, only upper limits were obtained for the high ionisation lines [O III] $53 \mu \mathrm{m},[\mathrm{O}$ III] $88 \mu \mathrm{m}$ and [N II] $122 \mu \mathrm{m}$. This is consistent with the luminosities derived by Larsson et al. (2000), indicating the presence of stellar sources generating at best only gentle UV-fields.

We can also exclude the presence of extended strongly shocked regions. For instance, if associated with the fast moving objects of Rodríguez et al. (1989), our data imply that the physical scales of these shocks would be small, $\ll 1^{\prime \prime}$ (e.g. for $v_{\mathrm{s}} \sim 200 \mathrm{~km} \mathrm{~s}^{-1}$ and $n_{0} \gtrsim 10^{5} \mathrm{~cm}^{-3}$; see: Cameron \& Liseau 1990; Liseau et al. 1996a).

We can conclude that the degree of ionisation of the atomic gas is generally low. Lines of low-ionisation species will be discussed in the next sections. 

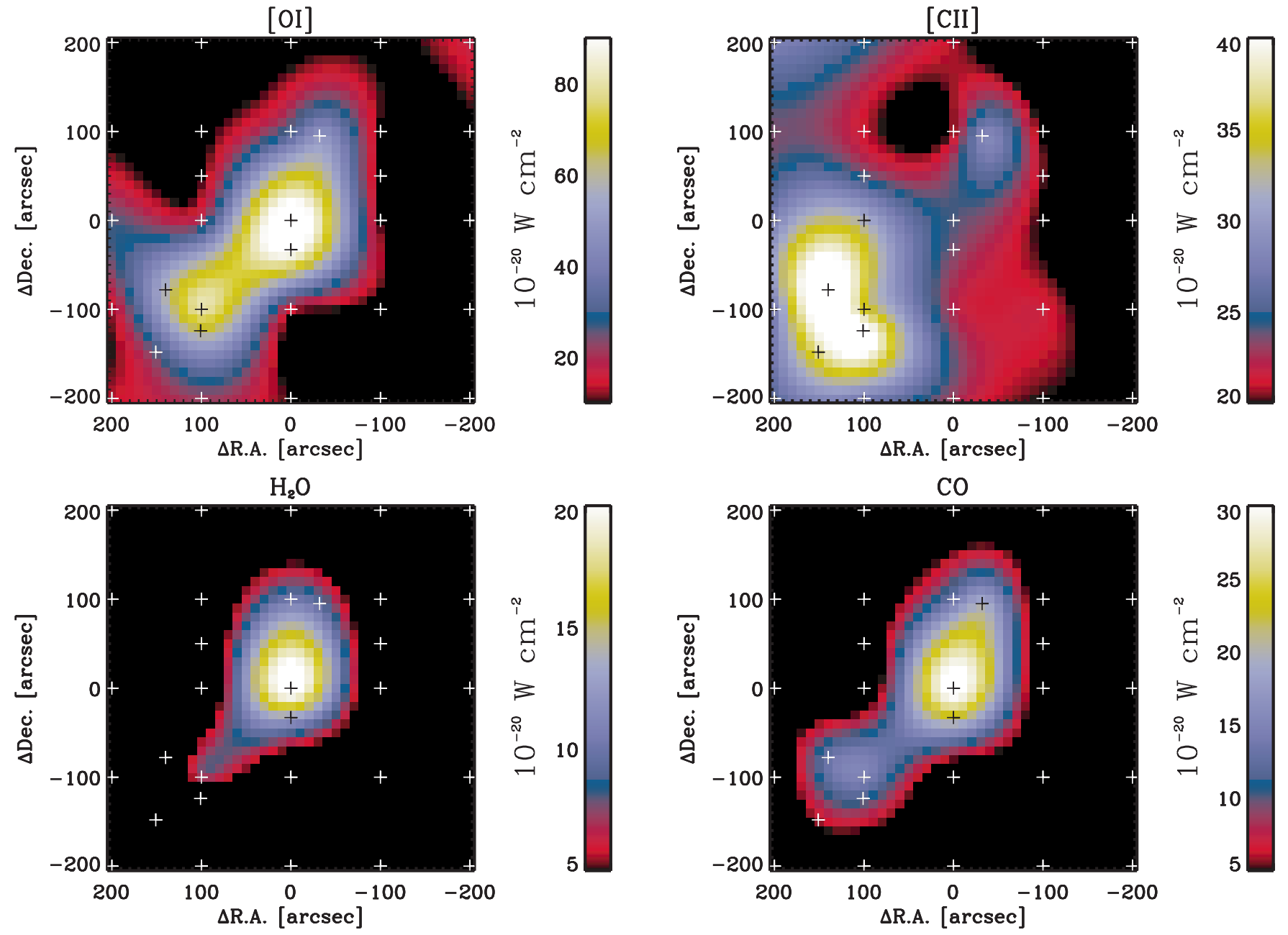

Fig. 3. Maps of integrated line fluxes of [O I] $63 \mu \mathrm{m},[\mathrm{C} \mathrm{II}] 157 \mu \mathrm{m}, \mathrm{H}_{2} \mathrm{O}\left(2_{12}-1_{01}\right)$ and $\mathrm{CO}(14-13)$, with individual flux levels indicated by the bars next to each frame. The crosses refer to the positions toward which the LWS spectra have been obtained.

Table 2. Line fluxes of atoms and ions toward SMM1.

\begin{tabular}{|c|c|c|c|c|c|c|c|c|c|}
\hline \multirow[t]{2}{*}{ Ion id } & \multirow[t]{2}{*}{ Transition } & \multicolumn{4}{|c|}{$\begin{array}{l}\text { Wavelength } \\
\qquad(\mu \mathrm{m})\end{array}$} & \multicolumn{2}{|c|}{$\begin{array}{c}\text { Flux } \times 10^{19} \\
\left(\mathrm{~W} \mathrm{\textrm {cm } ^ { - 2 }}\right)\end{array}$} & \multirow{2}{*}{$\begin{array}{c}\text { Single/ } \\
\text { Multi } \\
\text { fit }\end{array}$} & \multirow[t]{2}{*}{$\begin{array}{l}\text { LWS } \\
\text { Detector }\end{array}$} \\
\hline & & $\lambda$ & $\lambda_{\text {obs }}$ & $\Delta \lambda$ & $\sigma_{\lambda}$ & $F$ & $\Delta F$ & & \\
\hline \multirow[t]{2}{*}[\mathrm{CII}]{} & ${ }^{2} \mathrm{P}_{3 / 2}{ }^{2} \mathrm{P}_{1 / 2}$ & 157.74 & $157.72 \pm 0.02$ & -0.02 & $0.46 \pm 0.03$ & $2.07 \pm 0.21$ & 0.35 & $\mathrm{~S}$ & LW 4 \\
\hline & & & $157.72 \pm 0.04$ & -0.02 & 0.60 Fix & $2.31 \pm 0.20$ & 0.34 & $\mathrm{~S}$ & LW 4 \\
\hline \multirow[t]{6}{*}[\mathrm{OI}]{} & ${ }^{3} \mathrm{P}_{0}-{ }^{3} \mathrm{P}_{1}$ & 145.53 & 145.53 Fix & +0.00 & 0.60 Fix & $0.37 \pm 0.84$ & 0.90 & $\mathrm{M}$ & LW 4 \\
\hline & & & 145.53 Fix & +0.00 & 0.60 Fix & $0.76 \pm 0.83$ & 0.89 & M & LW 3 \\
\hline & ${ }^{3} \mathrm{P}_{1}-{ }^{3} \mathrm{P}_{2}$ & 63.18 & $63.19 \pm 0.01$ & +0.01 & $0.27 \pm 0.01$ & $9.5 \pm 0.7$ & 0.74 & $\mathrm{~S}$ & SW 3 \\
\hline & & & $63.19 \pm 0.01$ & +0.01 & 0.29 Fix & $9.8 \pm 0.4$ & 0.46 & $\mathrm{~S}$ & SW 3 \\
\hline & & & $63.17 \pm 0.01$ & -0.01 & $0.28 \pm 0.01$ & $10.3 \pm 0.9$ & 0.96 & $\mathrm{~S}$ & SW 2 \\
\hline & & & $63.17 \pm 0.01$ & -0.01 & 0.29 Fix & $10.5 \pm 0.5$ & 0.60 & $\mathrm{~S}$ & SW 2 \\
\hline$[\mathrm{NII}]$ & ${ }^{3} \mathrm{P}_{2}-{ }^{3} \mathrm{P}_{1}$ & 121.90 & & & & & 0.29 & $\mathrm{~S}$ & LW 2 \\
\hline \multirow[t]{3}{*}{ [OIII] } & ${ }^{3} \mathrm{P}_{1}-{ }^{3} \mathrm{P}_{0}$ & 88.36 & & & & & 0.47 & $\mathrm{~S}$ & LW 1 \\
\hline & & & & & & & 0.32 & $\mathrm{~S}$ & SW 5 \\
\hline & ${ }^{3} \mathrm{P}_{2}-{ }^{3} \mathrm{P}_{1}$ & 51.82 & & & & & 0.73 & $\mathrm{~S}$ & SW 2 \\
\hline
\end{tabular}

\subsubsection{Extended emission: [C $\mathrm{CI}] 157 \mu \mathrm{m}$ and [O I] $63 \mu \mathrm{m}$}

The spatial distribution of the [C II] $157 \mu \mathrm{m}$ emission is shown in Fig. 3 from which it is evident that the emission varies within a factor of about two. The S 68 nebulosity is pronounced in the $[\mathrm{C}$ II $] 157 \mu \mathrm{m}$ line, making it likely that its origin is from a photondominated region (PDR), close to the cloud surface.

This idea can be tested quantitatively by invoking also the observed [O I] $63 \mu \mathrm{m}$ emission. The LWS subtends a 


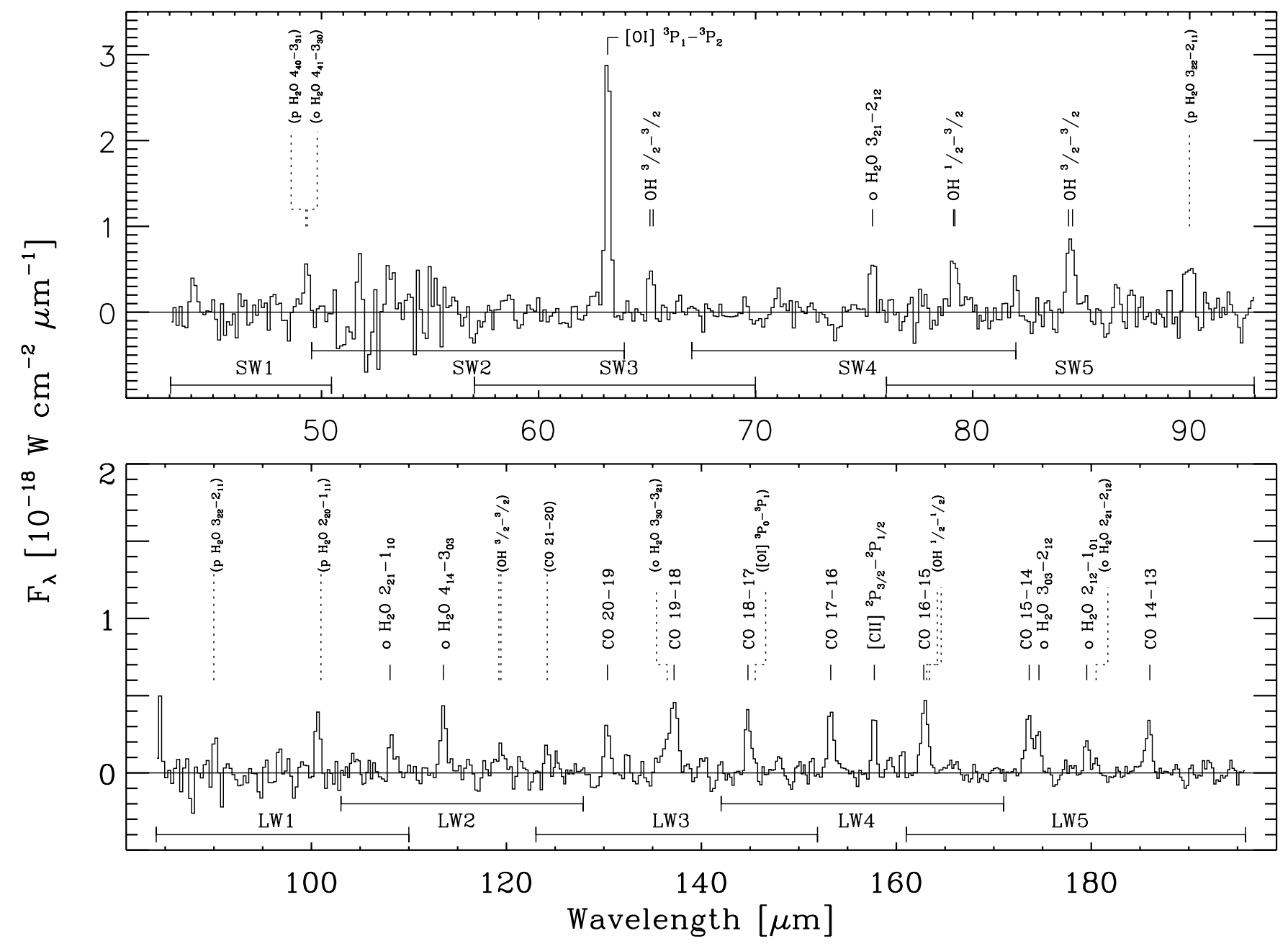

Fig. 4. The LWS spectrum (43 to $197 \mu \mathrm{m}$ ) of Serpens SMM 1 with line positions indicated. For clarity the strong continuum (Larsson et al. 2000) has been subtracted. The originally four times oversampled data have been rebinned to $0.1 \mu \mathrm{m}$ for the short wavelength (SW) and to $0.2 \mu \mathrm{m}$ for the long wavelength (LW) spectral range. Also shown, below the spectrum, is the extent of the individual LWS detectors.

solid angle $\Omega_{\mathrm{LWS}}=9.0 \times 10^{-8} \mathrm{sr}$. Disregarding for a moment the peak emission (see below), we find for unit beam filling the line intensities $I_{157}=2 \times 10^{-5} \mathrm{erg} \mathrm{cm}^{-2} \mathrm{~s}^{-1} \mathrm{sr}^{-1}$ and $I_{63}=1 \times 10^{-5} \mathrm{erg} \mathrm{cm}^{-2} \mathrm{~s}^{-1} \mathrm{sr}^{-1}$, respectively. Hence, the line ratio [O I] $63 \mu \mathrm{m} /[\mathrm{C} \mathrm{II}] 157 \mu \mathrm{m}$ is about 0.5 (Fig. 5). These data are consistent with PDR-emission, where an interstellar radiation field about 10 times as intense as that of the solar neighbourhood, i.e. $G_{0} \sim 10$, is impinging on the outer layers of a cloud with densities in the range $n(\mathrm{H})=(0.1-1) \times 10^{5} \mathrm{~cm}^{-3}$ (cf. Figs. 4 and 5 of Liseau et al. 1999). This estimate of the strength of the UV field is in reasonable agreement with the FIRbackground measured by IRAS and ISO (Larsson et al. $2000)$, which would imply $G_{0}=5-25$. In the advocated PDR model, the [O I $] 145 \mu \mathrm{m}$ line is fainter by two orders of magnitude than the [O I] $63 \mu \mathrm{m}$ line. The observed line ratio, [O I] $63 \mu \mathrm{m} /[\mathrm{O} \mathrm{I}] 145 \mu \mathrm{m}>12$, is clearly consistent with this prediction. Finally, no detectable emission from higher ionisation stages would be expected. The PDR model would offer therefore a satisfactory explanation for the observed fine structure line distribution over the map.
If this PDR emission is treated as a large scale background and subtracted from the maps, the resulting line ratio toward the peak (SMM 1) increases dramatically, viz. to $[\mathrm{O}$ I] $63 \mu \mathrm{m} /[\mathrm{C}$ II $] 157 \mu \mathrm{m}=15 \pm 5$. Such large ratios are generally not predicted by PDR models but are a common feature of $J$-shocks (Hollenbach \& McKee 1989). The residual [O I] $63 \mu \mathrm{m}$ flux corresponds to an observed intensity $I_{63}=1 \times 10^{-4} \mathrm{erg} \mathrm{cm} \mathrm{cm}^{-2} \mathrm{~s}^{-1} \mathrm{sr}^{-1}$, more than two orders of magnitude below that of $J$-shock models (see Sect. 4.1.3). Interpreted as a beam filling effect, this would imply the size of the shocked regions to be about $4^{\prime \prime}$ to $5^{\prime \prime}$.

\subsubsection{The $[\mathrm{O}$ I] $63 \mu \mathrm{m}$ emission toward $\mathrm{HH} 460$}

Toward the interstellar shock, HH 460, the [O I] $63 \mu \mathrm{m}$ flux is not conspicuously larger than that of [C II] $157 \mu \mathrm{m}$, as one might naively expect for shock excitation, and we cannot exclude the possibility that the spatial coincidence with the [O I] $63 \mu \mathrm{m}$ emission spot is merely accidental. However, pursuing the shock idea we find that, for the previously inferred cloud densities, $\gtrsim 10^{5} \mathrm{~cm}^{-3}$, 
Table 3. CO line measurements in the spectrum of SMM1.

\begin{tabular}{|c|c|c|c|c|c|c|c|c|}
\hline $\begin{array}{l}\text { Transition } \\
(J \rightarrow J-1)\end{array}$ & $\lambda$ & $\begin{array}{l}\text { Wavelength } \\
\qquad \begin{array}{c}(\mu \mathrm{m}) \\
\lambda_{\text {obs }}\end{array}\end{array}$ & $\Delta \lambda$ & $\begin{array}{c}\text { Width } \\
(\mu \mathrm{m}) \\
\sigma_{\lambda} \\
\end{array}$ & $\begin{array}{c}\text { Flux } \times 10^{19} \\
\left(\mathrm{~W} \mathrm{~cm}{ }^{-2}\right) \\
F \\
\end{array}$ & $\begin{array}{c}\mathrm{Err} \times 10^{19} \\
\left(\mathrm{~W} \mathrm{\textrm {cm } ^ { - 2 }}\right) \\
\Delta F\end{array}$ & $\begin{array}{l}\text { Single/ } \\
\text { Multi } \\
\text { fit }\end{array}$ & $\begin{array}{l}\text { LWS } \\
\text { Detector }\end{array}$ \\
\hline \multirow[t]{2}{*}{$14-13$} & 186.00 & $185.88 \pm 0.03$ & -0.12 & $0.76 \pm 0.03$ & $2.64 \pm 0.30$ & 0.37 & $\mathrm{~S}$ & LW 5 \\
\hline & & $185.90 \pm 0.03$ & -0.10 & 0.60 Fix & $\overline{2.32 \pm 0.16}$ & 0.27 & S & LW 5 \\
\hline \multirow[t]{3}{*}{$15-14$} & 173.63 & $173.60 \pm 0.03$ & -0.03 & $0.81 \pm 0.03$ & $3.34 \pm 0.28$ & 0.35 & S & LW 5 \\
\hline & & $173.59 \pm 0.03$ & -0.04 & 0.60 Fix & $2.81 \pm 0.21$ & 0.30 & $\mathrm{~S}$ & LW 5 \\
\hline & & $173.58 \pm 0.03$ & -0.05 & 0.60 Fix & $\underline{2.77 \pm 0.50}$ & 0.55 & M & LW 5 \\
\hline \multirow[t]{6}{*}{$16-15$} & 162.81 & $162.90 \pm 0.02$ & +0.09 & $0.98 \pm 0.02$ & $4.58 \pm 0.19$ & 0.28 & $\mathrm{~S}$ & LW 5 \\
\hline & & $162.92 \pm 0.04$ & +0.12 & 0.60 Fix & $3.38 \pm 0.34$ & 0.40 & $\mathrm{~S}$ & LW 5 \\
\hline & & 162.81 Fix & & 0.60 Fix & $2.67 \pm 0.50$ & 0.54 & M & LW 5 \\
\hline & & $162.93 \pm 0.02$ & +0.12 & $0.59 \pm 0.02$ & $\overline{3.19 \pm 0.18}$ & 0.36 & $\mathrm{~S}$ & LW 4 \\
\hline & & $162.93 \pm 0.04$ & +0.12 & 0.60 Fix & $3.21 \pm 0.10$ & 0.33 & $\mathrm{~S}$ & LW 4 \\
\hline & & 162.81 Fix & +0.00 & 0.60 Fix & $\underline{2.86 \pm 0.34}$ & 0.46 & M & LW 4 \\
\hline \multirow[t]{2}{*}{$17-16$} & 153.27 & $153.28 \pm 0.02$ & +0.01 & $0.64 \pm 0.02$ & $\overline{2.81 \pm 0.20}$ & 0.34 & S & LW 4 \\
\hline & & $153.27 \pm 0.02$ & +0.00 & 0.60 Fix & $\overline{2.71 \pm 0.11}$ & 0.30 & S & LW 4 \\
\hline \multirow[t]{6}{*}{$18-17$} & 144.78 & $144.73 \pm 0.02$ & -0.05 & $0.57 \pm 0.03$ & $2.55 \pm 0.31$ & 0.44 & S & LW 4 \\
\hline & & $144.75 \pm 0.03$ & -0.03 & 0.60 Fix & $2.63 \pm 0.16$ & 0.36 & $\mathrm{~S}$ & LW 4 \\
\hline & & 144.78 Fix & & 0.60 Fix & $\underline{2.50 \pm 0.22}$ & 0.39 & M & LW 4 \\
\hline & & $144.78 \pm 0.02$ & +0.00 & $0.50 \pm 0.02$ & $2.67 \pm 0.28$ & 0.42 & $\mathrm{~S}$ & LW 3 \\
\hline & & $144.79 \pm 0.03$ & +0.01 & 0.60 Fix & $\underline{2.94 \pm 0.23}$ & 0.38 & $\mathrm{~S}$ & LW 3 \\
\hline & & 144.78 Fix & & 0.60 Fix & $\overline{2.94 \pm 0.32}$ & 0.44 & M & LW 3 \\
\hline \multirow[t]{3}{*}{$19-18$} & 137.20 & $137.19 \pm 0.03$ & -0.01 & $0.80 \pm 0.04$ & $3.39 \pm 0.41$ & 0.53 & S & LW 3 \\
\hline & & $137.21 \pm 0.04$ & +0.01 & 0.60 Fix & $2.85 \pm 0.28$ & 0.43 & S & LW 3 \\
\hline & & 137.20 Fix & & 0.60 Fix & $\underline{2.83 \pm 0.30}$ & 0.45 & M & LW 3 \\
\hline \multirow[t]{2}{*}{$20-19$} & 130.37 & $130.35 \pm 0.03$ & -0.02 & $0.68 \pm 0.03$ & $\overline{2.48 \pm 0.32}$ & 0.48 & $\mathrm{~S}$ & LW 3 \\
\hline & & $130.34 \pm 0.03$ & -0.03 & 0.60 Fix & $\underline{2.33 \pm 0.18}$ & 0.39 & S & LW 3 \\
\hline \multirow[t]{4}{*}{$21-20$} & 124.19 & $124.21 \pm 0.05$ & +0.02 & $0.48 \pm 0.06$ & $\overline{0.98 \pm 0.32}$ & 0.48 & S & LW 3 \\
\hline & & $124.20 \pm 0.08$ & +0.01 & 0.60 Fix & $1.08 \pm 0.23$ & 0.42 & S & LW 3 \\
\hline & & $124.10 \pm 0.05$ & -0.09 & $0.52 \pm 0.06$ & $\overline{1.17 \pm 0.31}$ & 0.42 & S & LW 2 \\
\hline & & $124.10 \pm 0.06$ & -0.09 & 0.60 Fix & $\underline{1.25 \pm 0.18}$ & 0.34 & S & LW 2 \\
\hline $22-21$ & 118.58 & & & & & 0.29 & $\mathrm{~S}$ & LW 2 \\
\hline
\end{tabular}

Notes to the table:

$\Delta F=\sqrt{\sigma_{1}^{2}+\sigma_{\mathrm{c}}^{2}}$ where $\sigma_{\mathrm{l}}$ is the fitting error and $\sigma_{\mathrm{c}}$ is the rms-level of the surrounding continuum integrated over one spectral resolution element, i.e. $0.29 \mu \mathrm{m}$ and $0.60 \mu \mathrm{m}$ for the SW and LW detectors, respectively. $\mathrm{S}=$ Single line component fit; $\mathrm{M}=$ multi component line fit. Underlined flux values were used in the rotation diagram analysis and are shown in Fig. 7.

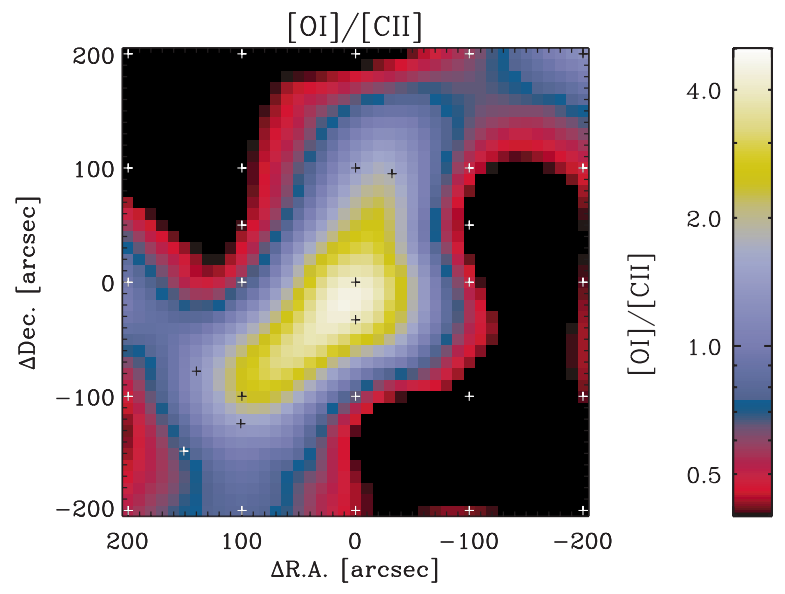

Fig. 5. Emission line intensity ratio map for [O I] $63 \mu \mathrm{m} /[\mathrm{C}$ II] $157 \mu \mathrm{m}$. Observed positions are identified by crosses. The observed flux distributions of the individual lines are shown in Fig. 3.

the $[\mathrm{O} \mathrm{I}] 63 \mu \mathrm{m}$ intensity is roughly constant with the shock speed (a few times $10^{-2} \mathrm{erg} \mathrm{cm}^{-2} \mathrm{~s}^{-1} \mathrm{sr}^{-1}$;
Hollenbach \& McKee 1989). These $J$-shock models do also predict that the accompanying [O I] $145 \mu \mathrm{m}$ emission would not be detectable in our observations and that any [C II] $157 \mu \mathrm{m}$ contribution would be totally insignificant.

The observed line intensity is $1 \times 10^{-5} \mathrm{erg} \mathrm{cm}^{-2} \mathrm{~s}^{-1}$ $\mathrm{sr}^{-1}$ which, if due to shock excitation, would indicate that the source fills merely a tiny fraction of the LWSbeam (beam dilution of $2.5 \times 10^{-4}$ ). A size of about $1^{\prime \prime}$ for the [O I] $63 \mu \mathrm{m}$ emitting regions of the $\mathrm{HH}$ object would thus be implied, which is comparable to the dimension of the dominating, point-like, optical knot HH $460 \mathrm{~A}$. From the observed line flux, a current mass loss rate from the HH-exciting source of $\dot{M}_{\text {loss }}=3 \times 10^{-7} M_{\odot} \mathrm{yr}^{-1}$ would be indicated (Hollenbach 1985; Liseau et al. 1997), which is at the $3 \%$ level of the mass accretion rate in the Serpens cloud core (Sect. 4.4).

Based on the $L_{63}-L_{\text {bol }}$ calibration by Liseau et al. (1997), one would predict that the luminosity of the central source is slightly less than $0.5 L_{\odot}$. No detailed information about the exciting source of $\mathrm{HH} 460$ is available, though. Based entirely on morphological arguments, 
Table 4. $\mathrm{H}_{2} \mathrm{O}$ line measurements in the spectrum of SMM 1.

\begin{tabular}{|c|c|c|c|c|c|c|c|c|}
\hline Transition & \multicolumn{3}{|c|}{$\begin{array}{l}\text { Wavelength } \\
\qquad(\mu \mathrm{m})\end{array}$} & $\begin{array}{c}\text { Width } \\
(\mu \mathrm{m}) \\
\sigma_{\lambda}\end{array}$ & $\begin{array}{c}\text { Flux } \times 10^{19} \\
\left(\mathrm{~W} \mathrm{~cm}{ }^{-2}\right) \\
F\end{array}$ & $\begin{array}{c}\text { Err } \times 10^{19} \\
\left(\mathrm{~W} \mathrm{~cm}^{-2}\right) \\
\Delta F\end{array}$ & $\begin{array}{l}\text { Single/ } \\
\text { Multi } \\
\text { fit }\end{array}$ & $\begin{array}{l}\text { LWS } \\
\text { Detector }\end{array}$ \\
\hline \multicolumn{9}{|l|}{ ortho- $\mathrm{H}_{2} \mathrm{O}$} \\
\hline \multirow[t]{2}{*}{$\overline{2_{21}-2_{12}}$} & 180.49 & $180.49 \pm 0.03$ & +0.00 & $0.45 \pm 0.03$ & $0.53 \pm 0.11$ & 0.24 & $\mathrm{~S}$ & LW 5 \\
\hline & & $180.49 \pm 0.05$ & +0.00 & 0.60 Fix & $0.60 \pm 0.01$ & 0.23 & $\mathrm{~S}$ & LW 5 \\
\hline \multirow[t]{2}{*}{$2_{12}-1_{01}$} & 179.53 & $179.55 \pm 0.04$ & +0.02 & $0.57 \pm 0.04$ & $1.40 \pm 0.24$ & 0.32 & $\mathrm{~S}$ & LW 5 \\
\hline & & $179.55 \pm 0.04$ & +0.02 & 0.60 Fix & $1.43 \pm 0.13$ & 0.25 & S & LW 5 \\
\hline \multirow[t]{3}{*}{$3_{03}-2_{12}$} & 174.63 & $174.63 \pm 0.03$ & +0.00 & $0.70 \pm 0.03$ & $2.15 \pm 0.24$ & 0.33 & $\mathrm{~S}$ & LW 5 \\
\hline & & $174.63 \pm 0.03$ & +0.00 & 0.60 Fix & $1.97 \pm 0.14$ & 0.26 & $\mathrm{~S}$ & LW 5 \\
\hline & & $174.63 \pm 0.06$ & +0.00 & 0.60 Fix & $1.93 \pm 0.66$ & 0.71 & $\mathrm{M}$ & LW 5 \\
\hline \multirow[t]{3}{*}{$3_{30}-3_{21}$} & 136.49 & $136.52 \pm 0.09$ & +0.03 & $0.64 \pm 0.11$ & $0.85 \pm 0.34$ & 0.48 & $\mathrm{~S}$ & LW 3 \\
\hline & & $136.51 \pm 0.06$ & +0.02 & 0.60 Fix & $0.81 \pm 0.11$ & 0.35 & $\mathrm{~S}$ & LW 3 \\
\hline & & 136.51 Fix & +0.02 & 0.60 Fix & $0.77 \pm 0.82$ & 0.88 & M & LW 3 \\
\hline \multirow[t]{2}{*}{$4_{14}-3_{03}$} & 113.54 & $113.54 \pm 0.04$ & +0.00 & $0.61 \pm 0.04$ & $2.96 \pm 0.39$ & 0.52 & $\mathrm{~S}$ & LW 2 \\
\hline & & $113.54 \pm 0.03$ & +0.00 & 0.60 Fix & $2.93 \pm 0.21$ & 0.41 & S & LW 2 \\
\hline \multirow[t]{4}{*}{$2_{21}-1_{10}$} & 108.07 & $108.13 \pm 0.05$ & +0.06 & $0.39 \pm 0.02$ & $1.29 \pm 0.40$ & 0.50 & S & LW 2 \\
\hline & & $108.18 \pm 0.07$ & +0.11 & 0.60 Fix & $1.58 \pm 0.31$ & 0.45 & $\mathrm{~S}$ & LW 2 \\
\hline & & $107.83 \pm 0.03$ & -0.25 & $0.51 \pm 0.05$ & $1.60 \pm 0.24$ & 0.52 & $\mathrm{~S}$ & LW 1 \\
\hline & & $107.84 \pm 0.05$ & -0.24 & 0.60 Fix & $1.79 \pm 0.22$ & 0.51 & S & LW 1 \\
\hline \multirow[t]{2}{*}{$3_{21}-2_{12}$} & 75.38 & $75.40 \pm 0.03$ & +0.02 & $0.29 \pm 0.03$ & $2.05 \pm 0.51$ & 0.60 & S & SW 4 \\
\hline & & $75.40 \pm 0.03$ & +0.02 & 0.29 Fix & $2.03 \pm 0.28$ & 0.42 & $\mathrm{~S}$ & SW 4 \\
\hline \multirow[t]{2}{*}{$4_{41}-330$} & 49.34 & $49.32 \pm 0.02$ & -0.02 & $0.25 \pm 0.02$ & $1.69 \pm 0.29$ & 0.37 & $\mathrm{~S}$ & SW 1 \\
\hline & & $49.32 \pm 0.02$ & -0.02 & 0.29 Fix & $1.81 \pm 0.18$ & 0.29 & M & SW 1 \\
\hline \multicolumn{9}{|l|}{ para- $\mathrm{H}_{2} \mathrm{O}$} \\
\hline \multirow[t]{2}{*}{$\frac{2_{20}-1_{11}}{2}$} & 100.98 & $100.66 \pm 0.05$ & -0.32 & $0.57 \pm 0.04$ & $2.53 \pm 0.68$ & 0.82 & S & LW 1 \\
\hline & & $100.66 \pm 0.06$ & -0.32 & 0.60 Fix & $2.60 \pm 0.37$ & 0.58 & $\mathrm{~S}$ & LW 1 \\
\hline \multirow[t]{2}{*}{$3_{22}-2_{11}$} & 89.99 & $89.99 \pm 0.05$ & +0.00 & $0.44 \pm 0.04$ & $2.71 \pm 0.76$ & 0.83 & $\mathrm{~S}$ & SW 4 \\
\hline & & $90.01 \pm 0.04$ & +0.02 & 0.29 Fix & $2.02 \pm 0.42$ & 0.53 & $\mathrm{~S}$ & SW 4 \\
\hline
\end{tabular}

Ziener \& Eislöffel (1999) associate HH 460 with SMM 1, and Davis et al. (1999) either with SMM1 or with SMM 9/S68N. The inferred luminosities of these objects, $71 L_{\odot}$ and $16 L_{\odot}$, respectively (Larsson et al. 2000), are however much larger than that inferred for the putative source driving HH 460. Evidently, the present status regarding the identification of the driving source of $\mathrm{HH} 460$ is inconclusive. Proper motion and radial velocity data would be helpful in this context.

\section{2. $\mathrm{H}_{2}, \mathrm{CO}, \mathrm{H}_{2} \mathrm{O}$ and $\mathrm{OH}$ toward SMM 1}

We can directly dismiss the PDR of Sect. 4.1.2 as responsible for the molecular line emission observed with the LWS, since gas densities and kinetic temperatures are far too low for any significant excitation of these transitions. Shock excitation would be an obvious option. In the following, we will examine the line spectra of $\mathrm{H}_{2}, \mathrm{CO}, \mathrm{H}_{2} \mathrm{O}$ and $\mathrm{OH}$.

\subsubsection{Rotation diagram: $\mathrm{H}_{2}$ and $\mathrm{CO}$}

The analytical technique known as "rotation diagram" analysis is relatively simple and easy to apply to wavelength integrated molecular rotational line data. The advantages and the shortcomings of this analysis tool have been thoroughly discussed by Goldsmith \& Langer (1999).

Assuming the lines to be optically thin and to be formed in Local Thermodynamic Equilibrium (LTE), one can derive the equation of a straight line for the molecular column density as a function of the upper level energy in temperature units. The slope of this line is the reciprocal excitation temperature of the levels (which in LTE is the same for all levels and equals the kinetic gas temperature), viz.

$\ln \left(\frac{4 \pi}{h \nu_{0} g_{\mathrm{u}} A_{\mathrm{ul}}} \frac{\int_{\text {line }} F_{\lambda} \mathrm{d} \lambda}{\Omega_{\text {beam }}}\right)=\ln \left(\frac{N_{\mathrm{mol}}}{Q\left(T_{\mathrm{ex}}\right)}\right)-\frac{E_{\mathrm{u}}}{k T_{\mathrm{ex}}}$

where the symbols have their usual meaning. The left hand side of Eq. (1) entails the column density of the molecules in the upper levels. For $\mathrm{H}_{2}$, the upper level energies, $E_{\mathrm{u}}$, were obtained from Abgrall \& Roueff (1989) and the Einstein transition probabilities, $A_{\mathrm{ul}}$, were adopted from Wolniewicz et al. (1998). For CO, these data were taken from Chandra et al. (1996). The statistical weights of the upper levels are given by $g_{\mathrm{u}}=(2 I+1)\left(2 J_{\mathrm{u}}+1\right)$, where $I$ is the quantum number of the nuclear spin. Further, for the evaluation of the approximate partition function

$Q\left(T_{\mathrm{ex}}\right) \approx \frac{k T_{\mathrm{ex}}}{h c B_{0}}$ 
Table 5. OH line measurements in the spectrum of SMM 1.

\begin{tabular}{|c|c|c|c|c|c|c|c|c|c|}
\hline \multicolumn{2}{|c|}{${ }^{2} \Pi$ Transition } & \multicolumn{4}{|c|}{$\begin{array}{l}\text { Wavelength } \\
\qquad(\mu \mathrm{m})\end{array}$} & \multirow{2}{*}{$\begin{array}{c}\text { Flux } \times 10^{19} \\
\left(\mathrm{~W} \mathrm{~cm}^{-2}\right) \\
F\end{array}$} & \multirow{2}{*}{$\begin{array}{c}\text { Err } \times 10^{19} \\
\left(\mathrm{~W} \mathrm{~cm}^{-2}\right) \\
\Delta F\end{array}$} & \multirow{2}{*}{$\begin{array}{c}\text { Single/ } \\
\text { Multi } \\
\text { fit }\end{array}$} & \multirow[t]{2}{*}{$\begin{array}{l}\text { LWS } \\
\text { Detector }\end{array}$} \\
\hline$\Omega-\Omega^{\prime}$ & $J-J^{\prime}$ & $\lambda$ & $\lambda_{\text {obs }}$ & $\Delta \lambda$ & $\sigma_{\lambda}$ & & & & \\
\hline \multirow[t]{2}{*}{$1 / 2-1 / 2$} & $3 / 2-1 / 2$ & 163.26 & 163.26 Fix & +0.00 & 0.60 Fix & $1.39 \pm 0.90$ & 0.93 & $\mathrm{~S}$ & LW 5 \\
\hline & & & 163.26 Fix & +0.00 & 0.60 Fix & $1.00 \pm 0.85$ & 0.91 & $\mathrm{~S}$ & LW 4 \\
\hline \multirow[t]{2}{*}{$3 / 2-3 / 2$} & $5 / 2-3 / 2$ & 119.34 & $119.38 \pm 0.20$ & +0.04 & $1.31 \pm 0.11$ & $2.07 \pm 0.49$ & 0.56 & $\mathrm{~S}$ & LW 2 \\
\hline & & & $119.42 \pm 0.07$ & +0.08 & 0.60 Fix & $1.28 \pm 0.22$ & 0.36 & $\mathrm{~S}$ & LW 2 \\
\hline \multirow[t]{4}{*}{$3 / 2-3 / 2$} & $7 / 2-5 / 2$ & 84.51 & $84.43 \pm 0.03$ & -0.08 & $0.39 \pm 0.03$ & $2.94 \pm 0.64$ & 0.79 & $\mathrm{~S}$ & LW 1 \\
\hline & & & $84.44 \pm 0.08$ & -0.07 & 0.60 Fix & $3.49 \pm 0.73$ & 0.87 & $\mathrm{~S}$ & LW 1 \\
\hline & & & $84.51 \pm 0.02$ & +0.00 & $0.41 \pm 0.02$ & $4.02 \pm 0.50$ & 0.58 & $\mathrm{~S}$ & SW 5 \\
\hline & & & $84.50 \pm 0.02$ & -0.01 & 0.29 Fix & $3.26 \pm 0.31$ & 0.42 & $\mathrm{~S}$ & SW 5 \\
\hline \multirow[t]{4}{*}{$1 / 2-3 / 2$} & $1 / 2-3 / 2$ & 79.15 & $79.14 \pm 0.02$ & -0.01 & $0.29 \pm 0.02$ & $2.84 \pm 0.38$ & 0.54 & $\mathrm{~S}$ & SW 5 \\
\hline & & & $79.14 \pm 0.02$ & -0.01 & 0.29 Fix & $2.86 \pm 0.21$ & 0.43 & $\mathrm{~S}$ & SW 5 \\
\hline & & & $79.17 \pm 0.07$ & +0.02 & $0.56 \pm 0.07$ & $2.22 \pm 0.80$ & 0.89 & $\mathrm{~S}$ & SW 4 \\
\hline & & & $79.14 \pm 0.04$ & -0.01 & 0.29 Fix & $1.47 \pm 0.34$ & 0.51 & $\mathrm{~S}$ & SW 4 \\
\hline \multirow[t]{2}{*}{$3 / 2-3 / 2$} & $9 / 2-7 / 2$ & 65.21 & $65.18 \pm 0.03$ & -0.03 & $0.24 \pm 0.03$ & $1.51 \pm 0.45$ & 0.47 & $\mathrm{~S}$ & SW 3 \\
\hline & & & $65.18 \pm 0.03$ & -0.03 & 0.29 Fix & $1.64 \pm 0.26$ & 0.30 & $\mathrm{~S}$ & SW 3 \\
\hline $3 / 2-3 / 2$ & $11 / 2-9 / 2$ & 53.30 & & & & & 0.73 & $\mathrm{~S}$ & SW 2 \\
\hline
\end{tabular}

Note to the table: $\lambda$ is an average wavelength for the various fine structure lines.

we used the rotational constant, $B_{0}=59.33451 \mathrm{~cm}^{-1}$, for $\mathrm{H}_{2}$ from Bragg et al. (1982). For CO, $B_{0}=1.9225 \mathrm{~cm}^{-1}$, was obtained from the data by Lovas et al. (1979). Graphs of Eq. (1) are shown in Figs. 6 and 7, fitted to the CAM-CVF and LWS data, respectively.

To obtain a consistent result, the $\mathrm{H}_{2}$ data need to be corrected for the foreground extinction. Using the data of Ossenkopf \& Henning (1994; model for thin ice coating, $n=10^{5} \mathrm{~cm}^{-3}, t=10^{5} \mathrm{yr}$ ), an extinction correction of $A_{\mathrm{V}}=4.5 \mathrm{mag}$ resulted in a total column density of warm $\mathrm{H}_{2}$ gas of $N\left(\mathrm{H}_{2}\right)=(3.7 \pm 0.8) \times 10^{18} \mathrm{~cm}^{-2}$. The rotation temperature is $T_{\mathrm{ex}}=(1130 \pm 60) \mathrm{K}$ and an ortho-to-para ratio (nuclear spin state population) of $o / p=3$ is consistent with these data.

In Fig. 7, ground-based $\mathrm{CO}$ data from the literature were added for lower lying transitions. Evidently, the high- $J$ distribution appears markedly different from that of the low- $J$ lines. If these latter lines were truly optically thin, they could originate in extended cloud gas, where $T_{\text {low }-J}=(62 \pm 6) \mathrm{K}$, of column density $N_{\text {low }-J}=(2.0 \pm 0.2) \times 10^{17} \mathrm{~cm}^{-2}$. Seemingly in contrast, the LWS data identify gas at a characteristic temperature of $T_{\text {high-J }}=(320 \pm 20) \mathrm{K}$, with an LTE-column density of $N_{\text {high-J }}=(1.9 \pm 0.2) \times 10^{15} \mathrm{~cm}^{-2}$.

These results are based on ad hoc assumptions, i.e. that of unit beam filling and of low optical depth in the lines, potentially underestimating the column densities, and that the level populations are distributed according to their LTE values. LTE may be a reasonably good assumption for the low- $J$ lines. Regarding CO, it is however questionable to what extent these are optically thin. On the other hand, low opacity may come close to the truth

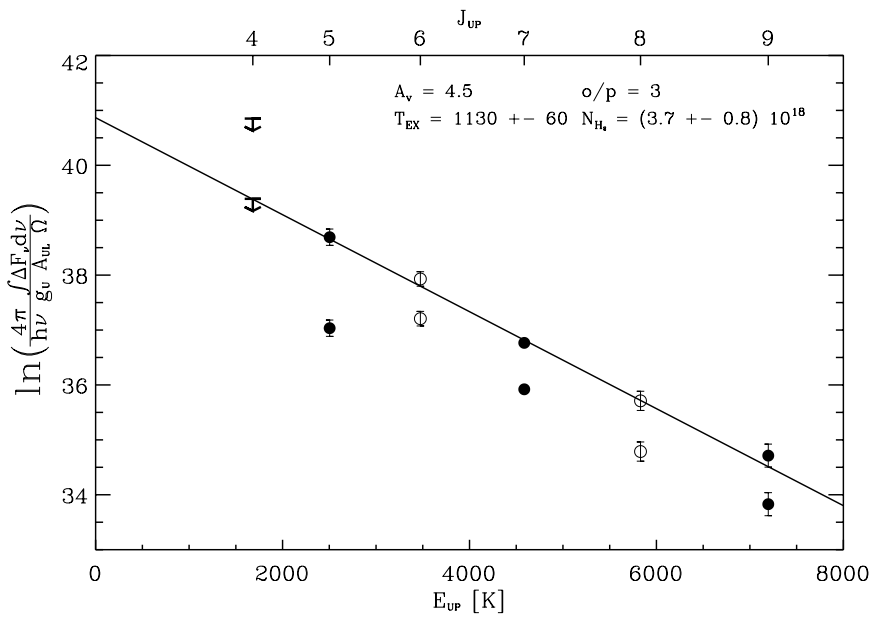

Fig. 6. Rotation diagram for the ortho- $\mathrm{H}_{2}$ (filled symbols) and para- $\mathrm{H}_{2}$ (open symbols and upper limit) lines observed toward the flow from SMM 1. A linear regression fit to extinction corrected data is shown by the full drawn line. The physical parameters with their formal errors are given in the figure (see also the text).

for the high- $J$ lines, but LTE is not at all guaranteed a priori for these transitions. Obviously, one needs to check how well these assumptions are justified. In the next sections, this will be addressed by employing first a method based on the Sobolev approximation and then a full Monte Carlo calculation, including gradients for both density and temperature. The latter method takes any (previously neglected) beam dilution effects directly into account. 


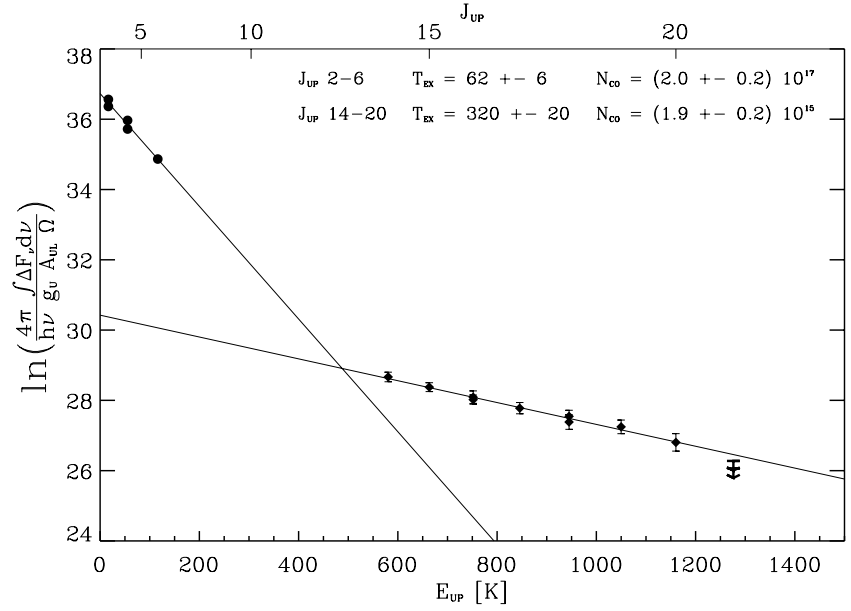

Fig. 7. Rotation diagram for the CO lines observed with the LWS (diamonds, this work) and for ground based data (filled circles), taken from Davis et al. (1999), Hogerheijde et al. (1999) and White et al. (1995). More than one LWS-value for the same upper energy $E_{\mathrm{u}} / k$ refer to measurements with different detectors. The data have been (ad hoc) fit by two linear segments, the solutions of which are given in the figure (see also the text).

\subsubsection{Large Velocity Gradient models: CO}

In the Large Velocity Gradient model (LVG) opacity effects in the lines are explicitly taken into account by introducing the photon escape probability formalism. Crudely speaking, the critical density of the transition, $n_{\text {crit }}=$ $A_{\mathrm{ul}} / C_{\mathrm{ul}}(T)$, can be lowered by means of an effective Einstein-probability, $A_{\mathrm{ul}} \beta_{\mathrm{esc}}$, where $\beta_{\mathrm{esc}}$ is in the range 0 to 1 for infinite and zero optical depth, respectively ${ }^{1}$. This can effectively "delay" line saturation. For illustrating purposes, $\beta_{\text {esc }} \sim 1 / \tau_{\text {line }}$ but, in general, $\beta_{\text {esc }}$ is geometry dependent (Castor 1970).

For under-resolved sources, an ambiguity can arise from the fact that hot and tenuous models may be indistinguishable from cool and dense ones. However, assuming that the rotation diagram analysis can provide an estimate of the kinetic gas temperature, LVG can be used to determine the average density of the emitting region. This is shown in Fig. 8, for the resulting $\log n\left(\mathrm{H}_{2}\right)=6.2 \pm 0.2 \mathrm{~cm}^{-3}$, which is in good agreement with the results by McMullin et al. (2000).

In these models, the presence of a diffuse radiation field is introduced by the dust temperature $T_{\text {dust }}=40 \mathrm{~K}$, the wavelength of unit optical depth $\lambda_{\tau=1}=200 \mu \mathrm{m}$, the frequency dependence of the dust emissivity $\beta=-1$ and a geometrical covering factor of 0.5 (cf. Larsson et al. 2000). The (clearly detected) high- $J$ lines are all only mildly sub-thermally excited (justifying a posteriori our initial assumption), but have substantial opacity, e.g. $\tau_{0(J=14-13)}=1.7$. First at $J_{\mathrm{u}}=22$ start the lines to become optically thin again $\left(\tau_{0(J=22-21)}=0.14\right)$.

\footnotetext{
1 The collision rate coefficients for $\mathrm{CO}, C_{i j}$, have been reevaluated and extended to higher $J$ values in Appendix B.
}

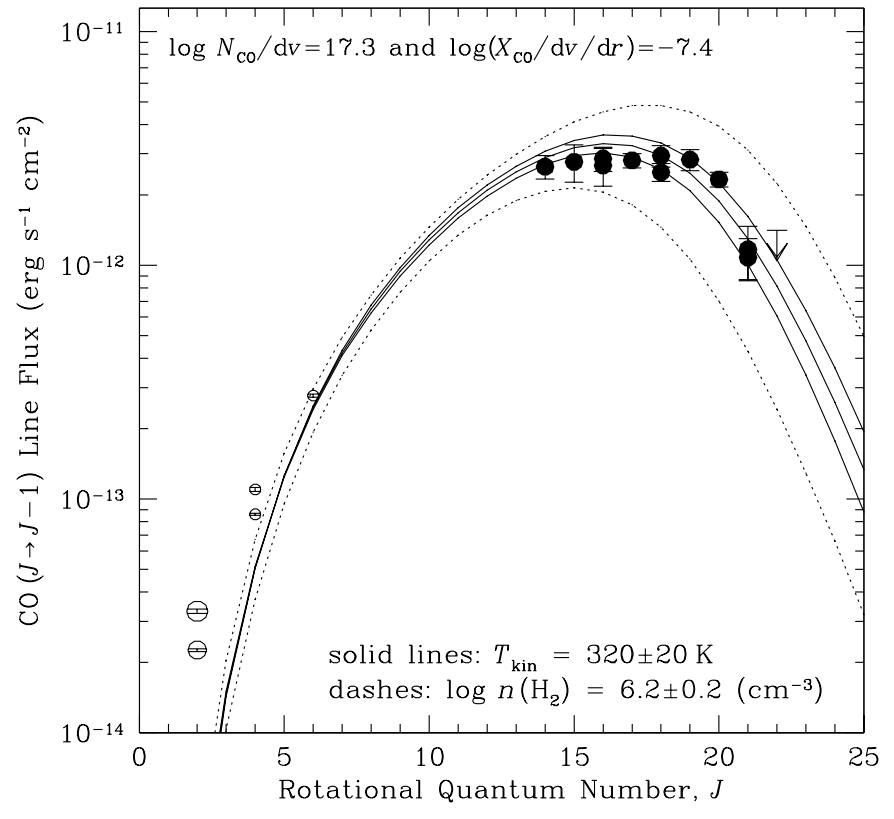

Fig. 8. The fluxes of rotational lines of $\mathrm{CO}$, observed with the LWS, are compared to LVG model computations. Filled circles with error bars refer to LWS data, where more than one value for a given $J$ correspond to different detectors. The upper limit is $3 \sigma$. In addition, open symbols represent ground based data, where the different sizes refer to different telecope beams (see Fig. 7). The physical parameters of the LVG model are indicated in the figure.

The principle parameter of the LVG model is related to the ratio of the column density to the line width, $N / \Delta v$. For a given density of the collision partners, $n\left(\mathrm{H}_{2}\right)$, this ratio is given by

$\frac{N_{\mathrm{CO}}}{\Delta v} \propto X_{\mathrm{CO}} / \frac{\partial v}{\partial r}$

where the right hand side is the "LVG-parameter". There, $\partial v / \partial r$ is the (Doppler) velocity gradient in the gas and $X_{\mathrm{CO}}$ is the molecular abundance relative to $\mathrm{H}_{2}$. From Eq. (3), it is clear that LVG models are, in general, not unique, since an increase of the column density could have the same effect as a decrease of the line width.

From the model fit, $N(\mathrm{CO})=1.5 \times 10^{18} \mathrm{~cm}^{-2}$ for the adopted $\Delta v=7.5 \mathrm{~km} \mathrm{~s}^{-1}$ (FWHM of a Gaussian line shape; see Sect. 4.3). A circular source would have a diameter of about $5^{\prime \prime}(1500 \mathrm{AU})$, a thickness of about $600 \mathrm{AU}$ and an $\mathrm{H}_{2}$ mass of about $0.01 M_{\odot}\left(\right.$ for $\left.X_{\mathrm{CO}}=10^{-4}\right)$. Finally, the total $\mathrm{CO}$ cooling rate amounts to $3.6 \times$ $10^{-1} L_{\odot}$.

The hot regions emitting in the $\mathrm{H}_{2}$ lines (Sect. 4.2.1) are not expected to contribute significantly to the CO emission observed with the LWS. We predict the strongest $\mathrm{CO}$ lines from this gas to be the $(J=4-3)$ and the $(J=5-4)$ transitions, with "LWS"-fluxes from a $10^{\prime \prime}$ source of about $1 \times 10^{-14} \mathrm{erg} \mathrm{cm}^{-2} \mathrm{~s}^{-1}$. 

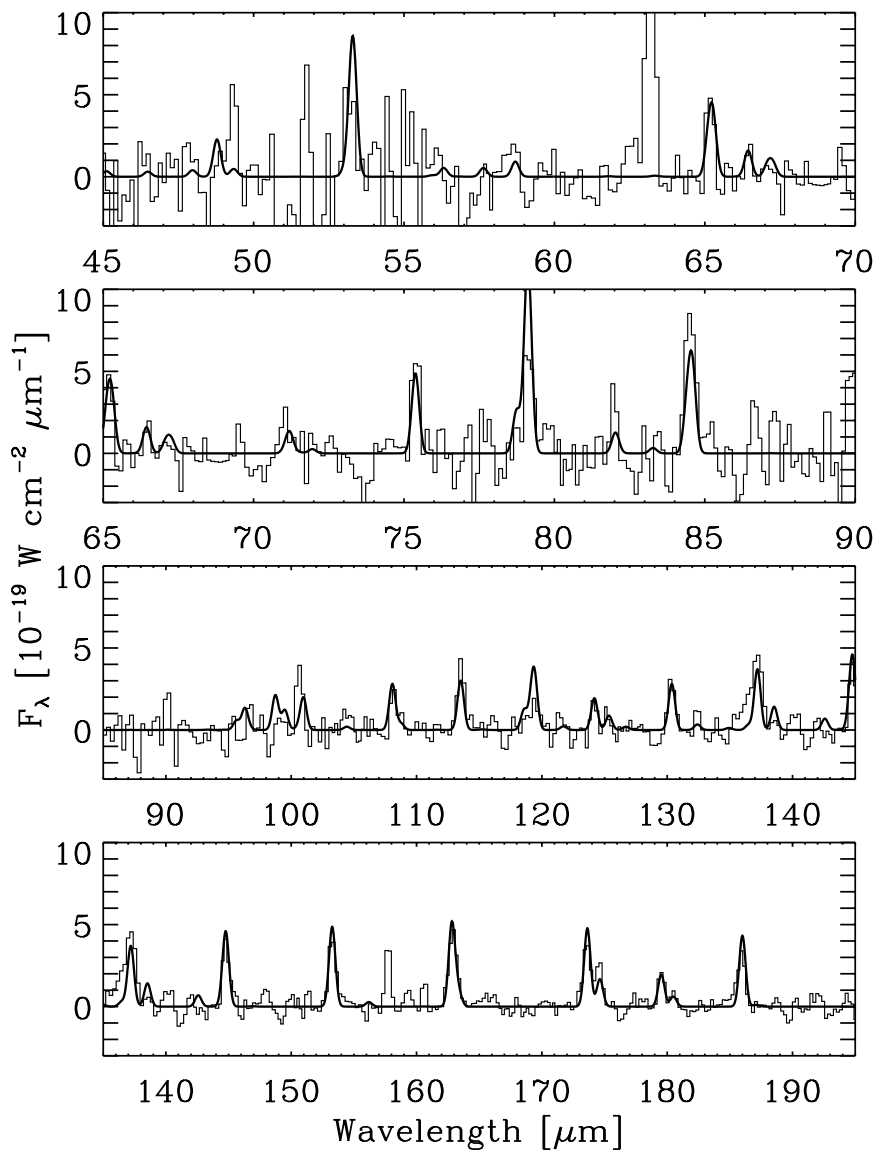

Fig. 9. The fit (smooth line) of the LVG model to the observed spectrum (histogram). All molecules, i.e. $\mathrm{CO},{ }^{13} \mathrm{CO}$, ortho$\mathrm{H}_{2} \mathrm{O}$, para- $\mathrm{H}_{2} \mathrm{O}$ and $\mathrm{OH}$, are assumed to share the same density and temperature, viz. $n\left(\mathrm{H}_{2}\right)=1.6 \times 10^{6} \mathrm{~cm}^{-3}, T_{\text {kin }}=320 \mathrm{~K}$; for further model details, see the text. Line identifications are as those given in Fig. 4.

\subsubsection{LVG models: ${ }^{13} \mathrm{CO}, \mathrm{H}_{2} \mathrm{O}$ and $\mathrm{OH}$}

The CO-model of the previous section can be used (by keeping $\partial v / \partial r$ constant) to investigate whether it is applicable also to other molecular species. Such a "one-sizefits-all" model would have the advantage of permitting the straightforward estimation of the relative abundance of these species (see Liseau et al. 1996b for an outline of this method). The reasonably satisfactory result of such computations for ${ }^{13} \mathrm{CO}$, ortho- $\mathrm{H}_{2} \mathrm{O}$, para- $\mathrm{H}_{2} \mathrm{O}$ and $\mathrm{OH}$ is presented in Fig. 9.

The ${ }^{13} \mathrm{CO}$ spectrum has been computed under the assumption that ${ }^{12} \mathrm{CO} /{ }^{13} \mathrm{CO}$ is as low as 40 (Leung \& Liszt 1976). The data are clearly consistent with this value, but the $\mathrm{S} / \mathrm{N}$ is insufficient to conclusively provide a better defined value. Since the ${ }^{13} \mathrm{CO}$ lines are all optically thin, the cooling in these lines $\left(1.3 \times 10^{-2} L_{\odot}\right)$ is relatively more efficient than that in $\mathrm{CO}$ (by almost a factor of two).

The $\mathrm{H}_{2} \mathrm{O}$ model is based on considering 45 levels for both ortho- and para- $\mathrm{H}_{2} \mathrm{O}$, including 164 transitions each. The radiative rates are from Chandra et al. (1984) and the scaled collision rates from Green et al. (1993). The model fit of the observed spectrum requires an $o / p=3$ for $\mathrm{H}_{2} \mathrm{O}$ and the derived $\mathrm{H}_{2} \mathrm{O}$-abundance is $X\left(\mathrm{H}_{2} \mathrm{O}\right)=1 \times 10^{-5}$. As expected, the excitation is sub-thermal and the lines are very optically thick (e.g., $\tau_{0(\circ 179.5 \mu \mathrm{m})}=433, \tau_{0(\mathrm{p} 101 \mu \mathrm{m})}=$ $218)$. Both the $380 \mathrm{GHz}$ ortho-transition $\left(4_{14}-3_{21}\right)$ and the $183 \mathrm{GHz}$ para-transition $\left(3_{13}-2_{20}\right)$ are predicted to be masing $\left(\tau_{0}=-1\right)$. The total cooling rate due to water vapour is $L\left(\mathrm{H}_{2} \mathrm{O}\right)=2.1 \times 10^{-1} L_{\odot}$, i.e. at the $60 \%$ level compared to the $\mathrm{CO}$ cooling rate.

For $\mathrm{OH}$, the Einstein $A$ values were computed from the data provided by D.Schwenke ${ }^{2}$, who also gives the energy levels. The collision rate coefficients for 50 transitions were obtained from Offer et al. (1994). As before, the excitation is sub-thermal and the lines are optically thick (e.g., $\tau_{0(119 \mu \mathrm{m})} \sim 170$ in each line of the doublet). This refers to the derived, relatively high, value of the $\mathrm{OH}$-abundance of $X(\mathrm{OH})=2 \times 10^{-6}\left(\mathrm{OH} / \mathrm{H}_{2} \mathrm{O}=\right.$ 0.2 ). The $\mathrm{OH}$ lines cool the gas as efficiently as $\mathrm{H}_{2} \mathrm{O}$, viz. $L(\mathrm{OH})=2.0 \times 10^{-1} L_{\odot}$. The model is overpredicting the $119 \mu \mathrm{m}$ line flux (whereas the $113 \mu \mathrm{m} \mathrm{H}_{2} \mathrm{O}$ line is underpredicted), perhaps indicating a distribution of temperatures (and densities). However, these lines fall in one of the least well performing LWS detectors (LW 2) and instrumental effects cannot be excluded.

So far, we have considered only models of a homogeneous source at a single kinetic temperature in a planeparallel geometry. The relaxation of these, likely unrealistic, assumptions is the topic of the next sections.

\subsection{4. $2 \mathrm{D}$ radiative transfer model of the dusty torus}

In our previous paper, we presented a self consistent radiative transfer model for the SED of SMM 1 (Larsson et al. 2000). For a simplified analysis and for a direct comparison with previous spherical models of the object, we adopted spherical geometry of the dusty envelope. The model provided a good fit to the observations longward of about $60 \mu \mathrm{m}$, but resulted in too low fluxes in the midIR. As already noted in that paper, the spherical symmetry may not be a very good assumption for SMM 1, the source driving the bipolar outflow. In this paper, we performed detailed modelling of the dusty object using our 2D radiative transfer code (Men'shchikov \& Henning 1997), which enabled us to quantitatively interpret existing dust continuum observations and to derive accurate physical parameters of SMM1. In the next section, the density and temperature structure of the model will be used in a Monte-Carlo calculation of the CO line radiation transfer in the envelope. Our approach and the model geometry are very similar to those for two other embedded protostars: HL Tau (Men'shchikov et al. 1999) and L1551 IRS 5 (White et al. 2000); we refer to the papers for more details on the general assumptions, computational aspects, and uncertainties of the modelling.

The model assumes that SMM 1 consists of an axiallysymmetric (quasi-toroidal), dense inner core surrounded by a similarly-shaped "envelope" (Fig. 10). A biconical

\footnotetext{
${ }^{2}$ Available at http://george.arc.nasa.gov:80/ dschwenke/
} 


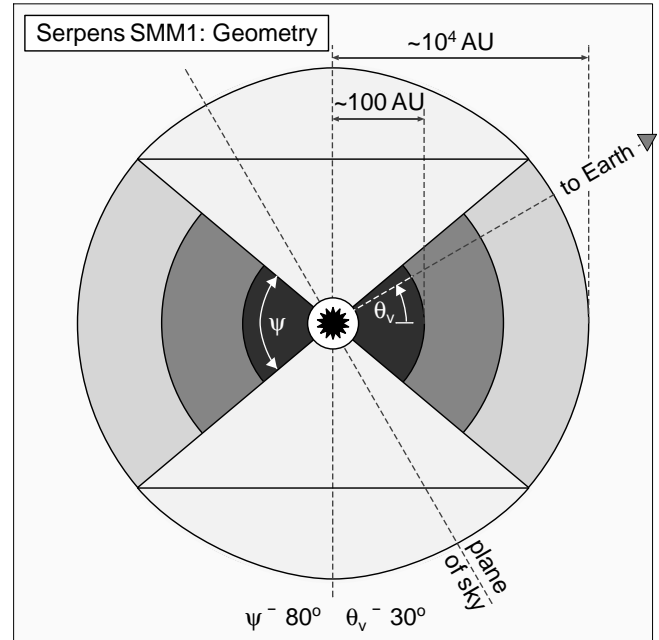

Fig. 10. Model geometry of the dusty torus of SMM 1 (see Sects. 4.2.4 and 4.2.5). Different shades of gray show schematically the density falling off outwards. The radius of the compact dense torus is $\sim 100 \mathrm{AU}$, whereas the outer radius of the envelope is based on our maps.

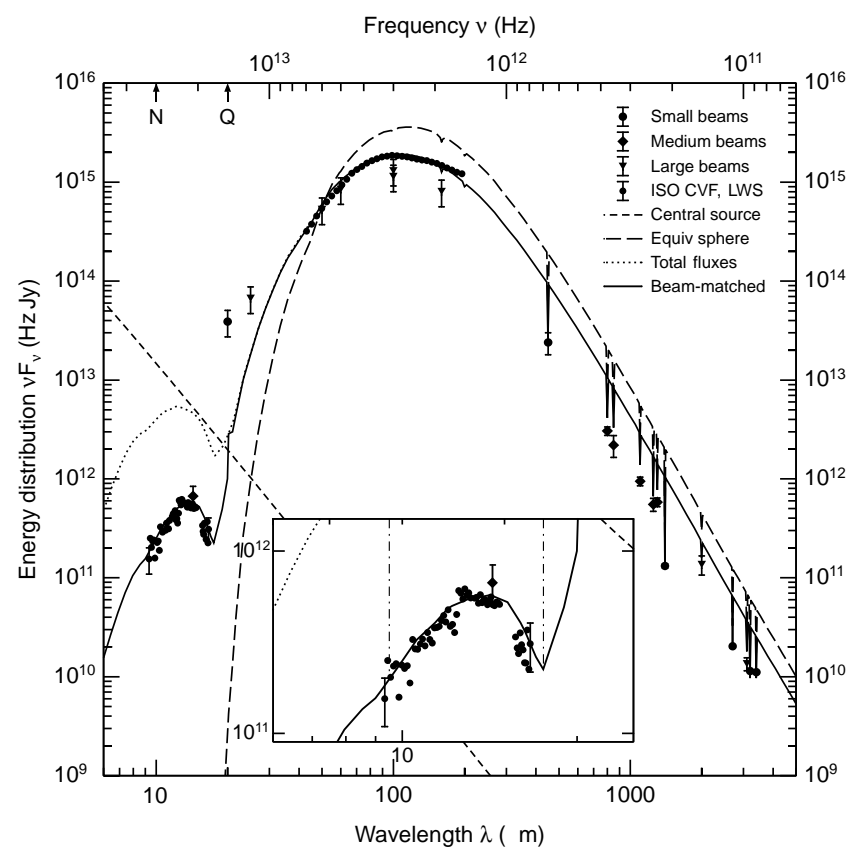

Fig. 11. Comparison of the observed SED of SMM 1 and the model of the dusty torus. The individual fluxes (see Larsson et al. 2000 for details) are labelled by different symbols, to distinguish between beams of different sizes. The model assumes that we observe the torus at an angle of $31^{\circ}$, relative to its midplane. The effect of beam sizes is shown by the vertical lines and by the difference between the dotted and solid lines in the model SED at mid-IR wavelengths. Whereas only the lower points of the vertical lines are relevant, we have connected them to the adjacent continuum by straight lines, to better visualise the effect. The SED for the equivalent spherical envelope is also shown, to illustrate the influence of the bipolar outflow cavities.

region of much lower density with a full opening angle of $100^{\circ}$ is presumed to be excavated in the otherwise
Table 6. Main input parameters of the dusty torus model.

\begin{tabular}{lc}
\hline \hline Parameter & Value \\
\hline Distance & $310 \mathrm{pc}$ \\
Central source luminosity & $140 L_{\odot}$ \\
Stellar effective temperature & $5000 \mathrm{~K}$ \\
Torus opening angle & $100^{\circ}$ \\
Viewing angle & $31^{\circ}$ \\
Torus dust melting radius & $2 \mathrm{AU}$ \\
Torus outer boundary & $1.4 \times 10^{4} \mathrm{AU}$ \\
Torus total mass (gas+dust) & $33 M_{\odot}$ \\
Density at melting radius & $2.5 \times 10^{-13} \mathrm{~g} \mathrm{~cm}^{-3}$ \\
Density at outer boundary & $1.4 \times 10^{-18} \mathrm{~g} \mathrm{~cm}^{-3}$ \\
Outflow visual $\tau_{\mathrm{v}}$ & 71 \\
Midplane $\tau_{\mathrm{v}}$ & 2200 \\
\hline
\end{tabular}

spherical envelope by the outflow from SMM 1. The structure, for brevity called "torus", is viewed at an inclination of $30^{\circ}$ with respect to the equatorial plane of the torus. Main input model parameters are summarised in Table 6.

As very little is known about dust properties in SMM 1, we adopted a dust model very similar to that applied by Men'shchikov \& Henning (1999) for HL Tau and by White et al. (2000) for L1551 IRS 5. The dust population consists of 4 components: (1) large dust particles of unspecified composition, with radii $100-6000 \mu \mathrm{m},(2)$ core-mantle grains made of silicate cores, covered by dirty ice mantles, (3) amorphous carbon grains, and (4) magnesium-iron oxide grains. The latter 3 components of dust grains have the same radii of $0.08-1 \mu \mathrm{m}$. The dust-to-gas mass ratios of the components are 0.01,0.0005, 0.0068, and 0.0005, respectively. The first component of very large grains is present only in the compact dense torus $(r \leq 120 \mathrm{AU})$, where all smaller grains are assumed to have grown into the large particles. Note that although unknown properties of dust generally introduce a major uncertainty in the derived model parameters, extremely high optical depths in SMM 1 make the model results not very sensitive to the specific choice of the grain properties, except for the presence of very large grains in the dense central core.

In the modelling of the dusty torus, we fitted all available photometry of SMM1, paying special attention to the effect of different beam sizes. Important constraints for the density structure were provided by the available submm and mm interferometry of the object. The model SED, compared to the observations in Fig. 11, fits almost every single individual flux in the entire range from the mid-IR to mm wavelengths. Note that it would be wrong to fit the observed data with the total model fluxes, because the angular size of SMM 1 is generally much larger than the photometric apertures. In fact, the model demonstrates that the effect of beam sizes on the fluxes may be as large as an order of magnitude. 

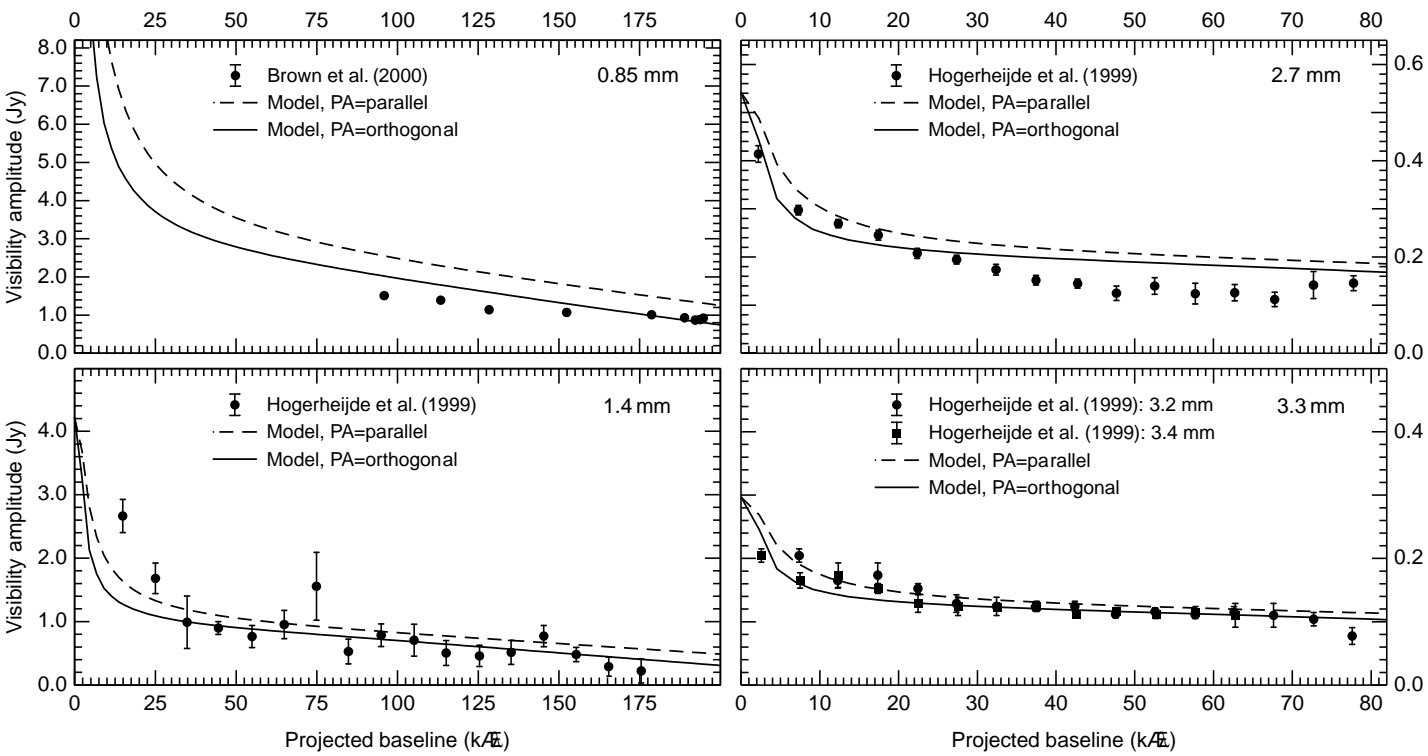

Fig. 12. Comparison of the model visibilities at $0.8 \mathrm{~mm}, 1.4 \mathrm{~mm}, 2.7 \mathrm{~mm}$, and $3.3 \mathrm{~mm}$ with available measurements of Brown et al. (2000) and Hogerheijde et al. (1999). The upper and lower curves in each panel show the visibilities for two directions in the plane of sky, parallel and orthogonal to the projected axis of the model.
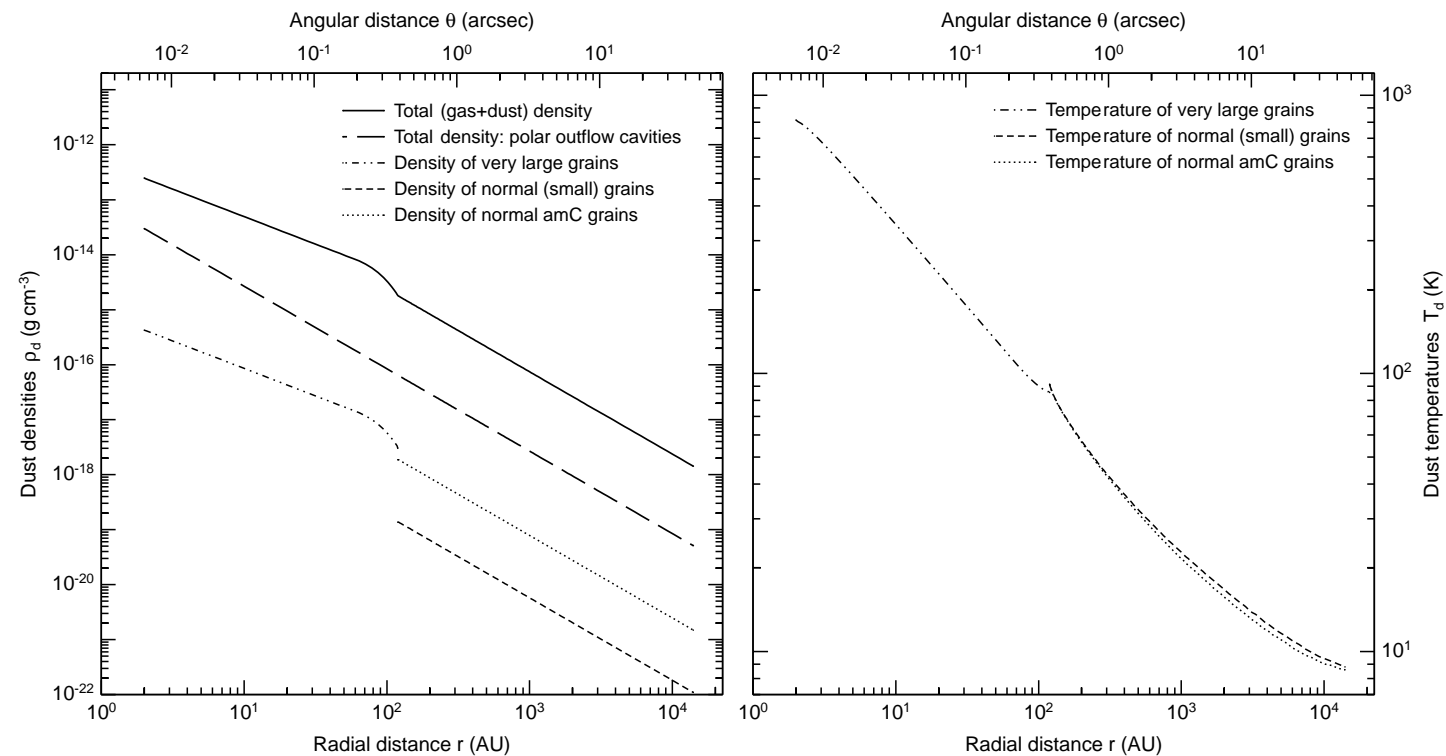

Fig. 13. Density and temperature structure of the model torus of SMM 1. Left panel: the total densities in the midplane and in polar outflow regions, and dust densities of different dust grain components (for only their smallest sizes). Right panel: the temperature profiles correspond to the midplane of the torus; they were obtained self-consistently from the equation of radiative equilibrium.

Comparison of the model visibilities to the interferometry data shown in Fig. 12 demonstrates that the model is also consistent with the observed spatial distribution of intensity. The visibilities indicate that there is a dense core inside of a lower density envelope. The radial density and temperature profiles of the model, are shown in Fig. 13. The innermost dense core has a $\rho \propto r^{-1}$ density gradient in the model, whereas the outer parts of the lower-density envelope have a steeper density distribution $\left(\rho \propto r^{-1.5}\right)$. The temperature distribution was obtained in iterations as a solution of the energy balance equation.

\subsection{5. $2 \mathrm{D}$ radiative transfer of the molecular emission}

We have used the density and temperature distributions of this dusty torus model in combination with a Monte Carlo scheme to compute the radiative transfer of the CO lines, and its isotopomers, through the source.

Observations of the Serpens cloud core in the $J=2-1$ transitions of the $\mathrm{CO}$-isotopomers $\mathrm{C}^{18} \mathrm{O}$ and $\mathrm{C}^{17} \mathrm{O}$ are present in the archive of the James Clerk Maxwell Telescope (JCMT). These potentially optically thin lines could trace the embedded core SMM1. Our disk model 

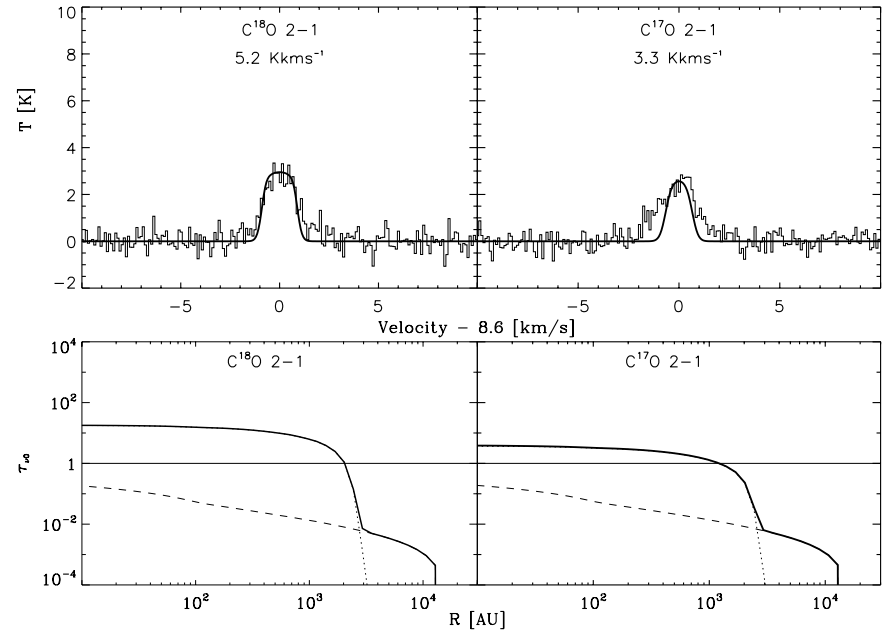

Fig. 14. Upper panel: for background emission corrected line profiles of low- $J$ CO isotopomers, viz. $\mathrm{C}^{18} \mathrm{O}(2-1)$ and $\mathrm{C}^{17} \mathrm{O}(2-1)$, toward SMM 1 are shown as histograms. The observations were retrieved from the JCMT archive. The results from 2D-Monte Carlo radiative transfer calculations for the disk/torus model are shown by smooth lines. The shown integrated line intensity refers to the model, for which adopted abundances are ${ }^{12} \mathrm{CO}: \mathrm{C}^{18} \mathrm{O}: \mathrm{C}^{17} \mathrm{O}=1: 550: 2750$ with $X\left({ }^{12} \mathrm{CO}\right)=10^{-4}$, and freeze-out of the molecules is treated in accordance with the work by Sandford \& Allamandola (1993). Lower panel: the total line centre optical depth in $\mathrm{C}^{18} \mathrm{O}(2-1)$ and $\mathrm{C}^{17} \mathrm{O}(2-1)$, along the line-of-sight toward the central region of the source, is shown by the solid line. Similarly, the dotted lines display the line opacities and the broken lines the dust opacities.

reproduces the observed line intensities of these low- $J$ isotopomers fairly well (Fig. 14). There, the averaged background emission of the surrounding gas has been subtracted, in order to reveal the line profiles of SMM 1 itself. From the figure it is evident that the $\mathrm{C}^{18} \mathrm{O}$ and $\mathrm{C}^{17} \mathrm{O}$ lines are optically thick out to a point, where the temperature falls below $15 \mathrm{~K}$ and where substantial condensation of the CO gas onto dust grains occurs. This CO freeze-out was treated following Sandford \& Allamandola (1993 and references therein), where the ice-to-gas ratio $\eta$ is proportional to the dust density $n$, and to functions of the gas and dust temperatures, $T_{\mathrm{g}}$ and $T_{\mathrm{d}}$ respectively, viz.

$\eta \propto n T_{\mathrm{g}}^{1 / 2} e^{1 / T_{\mathrm{d}}}$.

In the inner ("core") regions, line opacities are very high so that the photons are essentially trapped. Therefore, the excitation temperature of the molecules follows the kinetic temperature of the $\mathrm{H}_{2}$ gas. Beyond the core, i.e. in the "torus", $\tau$ drops quickly below unity for the high- $J$ lines, but there, the dust starts to contribute significantly to the (small) opacity (Fig. 15).

The CO lines falling into the LWS range are all formed in the inner, hotter parts of the source $\left(r \ll 10^{3} \mathrm{AU}\right.$, $\left.T \gg 10^{2} \mathrm{~K}\right)$. This small line forming region is insufficient to produce the observed flux levels, i.e. the model underpredicts observed high- $J$ line fluxes by more than two orders of magnitude. Irrespective of the geometry,
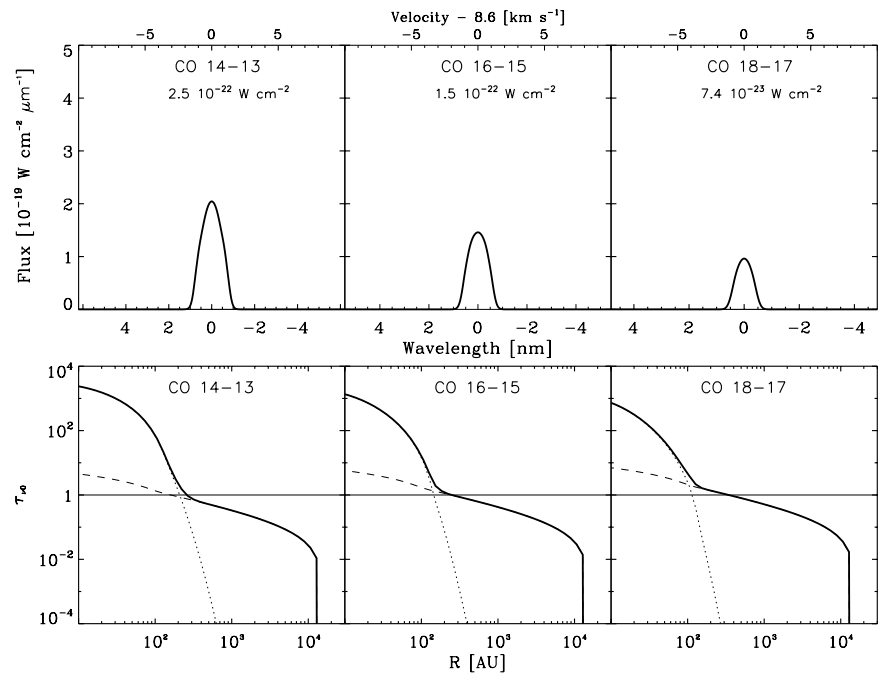

Fig. 15. Same as in Fig. 14, but for three high- $J$ CO transitions, which fall in the ISO-LWS spectral band.

we can conclude quite generally that the $\mathrm{CO}$ lines observed with the LWS do not originate from the central regions of SMM 1, be it an accretion disk, be it infalling gas (we have also computed "inside-out" collapse models).

For the excitation of this gas we need to consider alternative mechanisms and, since outflows are known to exist in this region, shock heating of the gas offers a natural option. Our temperature determinations for the molecular gas (Sect.4.2.1) are also consistent with this idea.

\subsection{Shock heating}

From the discussion of the preceding sections we can conclude that the heating of the gas is most likely achieved through shocks. These shocks are generated by flows within the LWS beam. Comparing the observed and predicted molecular line emission with the $J$-shock models by Hollenbach et al. (1989) and Neufeld \& Hollenbach (1994), we find that these models are in conflict with our observations.

In Fig. 16, we compare our observations of rotational lines of $\mathrm{H}_{2}, \mathrm{CO}, \mathrm{H}_{2} \mathrm{O}$ and $\mathrm{OH}$ with predictions of the $C$ shock models by Kaufman \& Neufeld (1996). The models for $\log n_{0}=5.5\left(\mathrm{~cm}^{-3}\right)$ and $v_{\mathrm{s}} \sim 15-20 \mathrm{~km} \mathrm{~s}^{-1}$ are in reasonable agreement with the for extinction corrected $\left(A_{\mathrm{V}}=\right.$ $12 \mathrm{mag}$ ) observed values for $\mathrm{H}_{2}$ and for a flux from $6^{\prime \prime} \times 6^{\prime \prime}$ (1 CVF-pixel). For CO, the model fits the observations for an adopted circular source of diameter $11^{\prime \prime}$. To achieve agreement for $\mathrm{H}_{2} \mathrm{O}$, the model fluxes would need to be adjusted downwards by a factor of 2.5, whereas an increase by more than one order of magnitude (a factor of 12) would be required for $\mathrm{OH}$. Evidently, $\mathrm{OH}$ is largely underproduced by these models, a fact also pointed out by Wardle (1999). If on the other hand the Wardle model is essentially correct, this would suggest that the ionisation rate in the Serpens cloud core is significantly higher (up to $10^{-15} \mathrm{~s}^{-1}$ ) than on the average in dark clouds, 
$\zeta=\left(10^{-18}-10^{-17}\right) \mathrm{s}^{-1}$. High X-ray activity is known to be present within the Serpens cloud core (Smith et al. 1999 and references therein). It is conceivable that such a high ionisation rate could also have considerable consequences for the cloud chemistry and its evolution. For instance, a relatively higher $\mathrm{H}_{3}^{+}$abundance could be expected, the effects of which (in addition to the enhanced abundance of $\mathrm{OH}$ ) may in fact have already been observed (e.g., HCN/HNC 1; McMullin et al. 2000 and references therein).

\subsection{Summarising discussion}

Based on their 0.8 mm JCMT-CSO interferometry, Brown et al. (2000) obtained estimates of the size, mass and average (dust) temperature of the disk of SMM 1. The estimated mass is larger and the size of the disk is smaller by one order of magnitude than what is required to account for the observed level of line emission (Sect. 4.2.2). Unless the disk (extended atmosphere?) is heated to very much higher temperatures (by an as yet to be identified mechanism) than the $60 \mathrm{~K}$ determined by Brown et al., we find it unlikely that the molecular line spectrum of SMM 1 is of circumstellar disk origin. Our own calculations (Sect. 4.2.4) confirm this conclusion.

It is intriguing that the luminosity of the spherical model of SMM1 $\left(71 L_{\odot}\right.$, Larsson et al. 2000) is close to the "magic number" of the classical main accretion phase of solar mass stars (Shu et al. 1987). At the elevated cloud temperature of the Serpens cloud core ( $\sim 40 \mathrm{~K}$, White et al. 1995), the isothermal sound speed is $0.4 \mathrm{~km} \mathrm{~s}^{-1}$ and, hence, the (time averaged, cf. Winkler \& Newman 1980) mass accretion rate corresponds roughly to $\dot{M}_{\text {acc }}=10^{-5} M_{\odot} \mathrm{yr}^{-1}$, yielding $L_{\text {acc }}=70 L_{\odot}$, where we have used the mass-radius relationship of Palla \& Stahler (1990). In this scenario, the age of SMM 1 would be about $10^{5} \mathrm{yr}$ or less, depending on the details of the acquired mass of the (presumably deuterium burning) central core. Regarding the data presented in this paper, we find it however difficult to reconcile this accretion shock model with our observations. As concluded in Sect. 4.2.4, the excitation of the observed lines requires significantly larger volumes at elevated densities and temperatures.

The $\mathrm{H}_{2}$ observations are partially resolved and there exists no ambiguity as to where, with respect to SMM 1, the emission arises (cf. Fig. 2). These lines trace a collimated outflow toward the northwest of SMM1, which is also seen in ro-vibrationally excited $\mathrm{H}_{2}$ line emission (Eiroa \& Casali 1989; Hodapp 1999). In the graphs of Fig. 16, we have assumed that also the LWS lines originate essentially at the location of the $\mathrm{H}_{2}$ spots (i.e. we have artificially introduced another factor of two for the fluxes). However, the dereddened data of Eiroa \& Casali (with the $A_{\mathrm{V}}$-value determined in Sect. 4.2.1) could potentially present an additional difficulty for the $C$-shock model (Kaufman \& Neufeld 1996). The estimated 1-0S(1) line intensity would in this case be larger by more than two
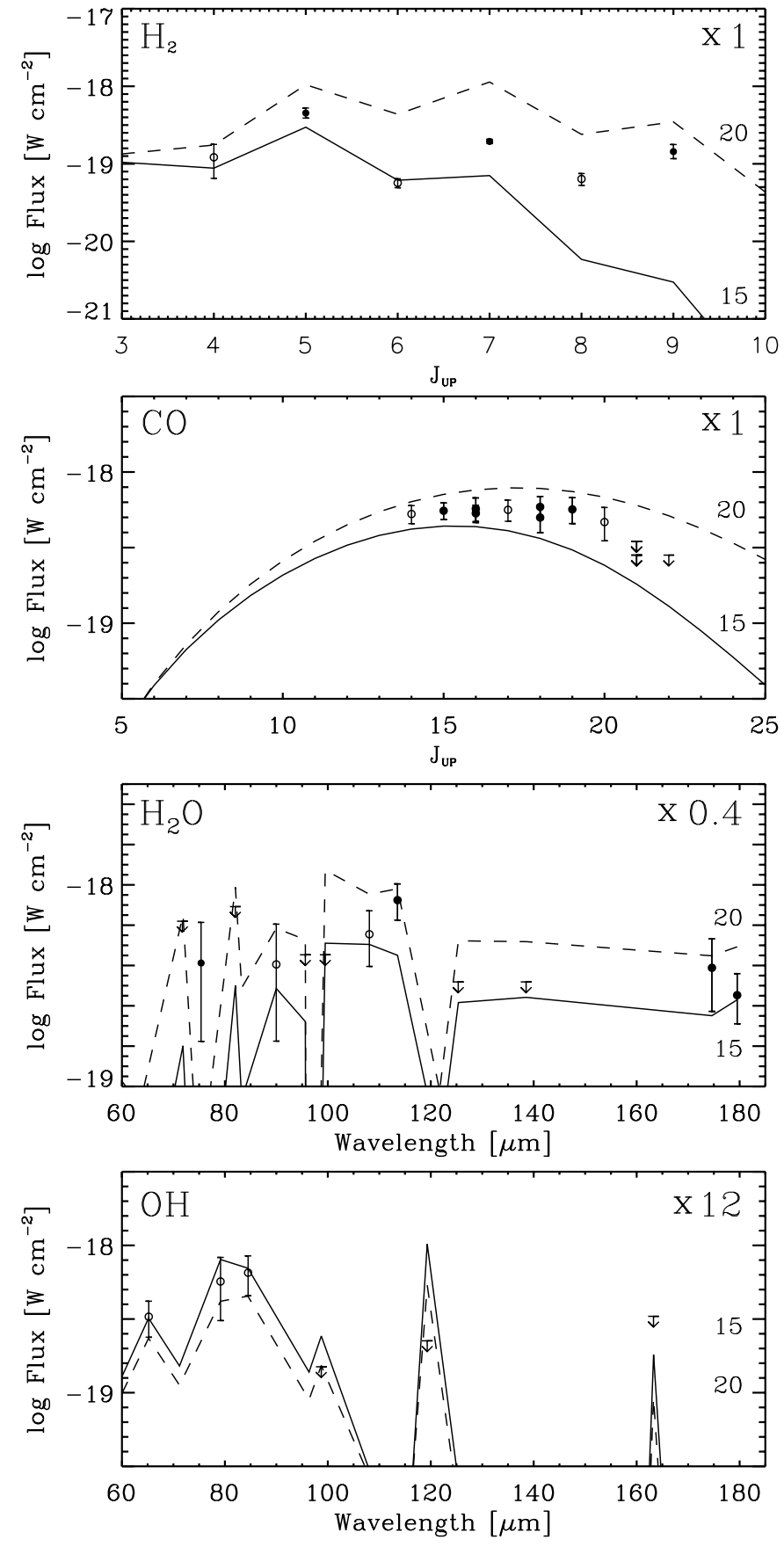

Fig. 16. Comparison of our molecular line observations with the predictions of theoretical models of $C$-shocks (Kaufman $\&$ Neufeld 1996). The pre-shock density is always $\log n_{0}=$ $5.5\left(\mathrm{~cm}^{-3}\right)$. Solid and dashed lines are for models with $v_{\mathrm{s}}=15 \mathrm{~km} \mathrm{~s}^{-1}$ and $20 \mathrm{~km} \mathrm{~s}^{-1}$, respectively. The $\mathrm{H}_{2}$ models refer to the CVF pixel size, whereas the other panels are for a circular source of diameter $11^{\prime \prime}$. The scaling factors, necessary to bring the observed and model data into agreement, are indicated in each frame by " $\times$ factor".

orders of magnitude than that predicted by the model. We cannot exclude at present, however, the possibility that the 1-0S(1) emission observed by Eiroa \& Casali (1989) is essentially unextinguished. Photometrically calibrated 
data at higher spatial resolution would be required to settle this issue.

The mechanical energy input by the flow is $L_{\text {mech }}=$ $0.5 \mu_{\text {gas }} m_{\mathrm{H}} n_{0} v_{\mathrm{s}}^{3} \times$ area, which for a pre-shock density of $\log n_{0}=5.5$, a shock velocity $v_{\mathrm{s}}=(15-20) \mathrm{km} \mathrm{s}^{-1}$, and a $5^{\prime \prime}$ source size yields $L_{\text {mech }}=(6.4-15.1) \times 10^{-2} L_{\odot}$. From the Kaufman \& Neufeld (1996) $C$-shock model, this gas is cooled by $\mathrm{H}_{2}$ at a rate of $(1.0-4.7) \times 10^{-2} L_{\odot}$. From the LWS data, we inferred the total cooling rate through the lines of $\mathrm{CO},{ }^{13} \mathrm{CO}, \mathrm{H}_{2} \mathrm{O}$ and $\mathrm{OH}$ of $78 \times 10^{-2} \mathrm{~L}_{\odot}$ (Sects. 4.2.2 and 4.2.3), corresponding to $0.5 \%$ to $1 \%$ of the total dust luminosity. This is larger by factors of 5 to 12 and it is thus not excluded that the shocked regions observed in the $\mathrm{H}_{2}$ lines and those giving rise to the FIR lines are not the same. We reached the same conclusion on the basis of our excitation and radiative transfer calculations.

The observed and background-corrected [O I] $63 \mu \mathrm{m}$ emission toward SMM 1 suggests a contribution also by $J$-shocks within the LWS beam (Sect. 4.1.2). Intriguingly, the derived dimensions are practically identical to those determined for the LWS-molecular emission, albeit existing $J$-shock models do not predict the relative intensities correctly. At present, we can merely conclude that shocks, in general, provide a plausible energy input mechanism, although the details of the shock type(s) are less clear. We propose that predominantly slow shock waves in the dense medium surrounding SMM 1 provide the heating of the molecules we have observed with ISO, whereas dynamical collapse is not directly revealed by our data.

\section{Conclusions}

Based on spectrophotometric ISO imaging with the LWS and the CAM-CVF of significant parts of the active star forming Serpens cloud core our main conclusions can be summarised as follows:

- We find the emission in the $[\mathrm{O}$ I $] 63 \mu \mathrm{m}$ and [C II] $157 \mu \mathrm{m}$ fine structure lines to be extended in our $8^{\prime} \times 8^{\prime}$ map. The absolute intensities and their ratios can be explained in terms of PDR models, where a UV field of $G_{0}=15 \pm 10$ is falling onto the outer layers of the dark cloud, where densities are of the order of $\left(10^{4}-10^{5}\right) \mathrm{cm}^{-3}$.

- Also the emission in rotational lines of $\mathrm{H}_{2} \mathrm{O}$ and high$J$ CO appears (slightly) extended, but we cannot exclude the possibility that it arises from point sources in the field, viz. the Class 0 objects SMM 9/S68 and SMM 4. The maximum of the emission is observed toward SMM 1, the dominating far infrared and submm source in the Serpens cloud core.

- The spectrum of SMM1 contains numerous lines of $\mathrm{CO}, \mathrm{H}_{2} \mathrm{O}$ and $\mathrm{OH}$. These lines are generally subthermally excited and optically thick and trace regions of dimensions $\sim 10^{3} \mathrm{AU}(\sqrt{1500 \times 600} \mathrm{AU})$, where temperatures are above $300 \mathrm{~K}$ and densities above $10^{6} \mathrm{~cm}^{-3}$. The derived abundances, relative to $\mathrm{H}_{2}$, are for $\mathrm{CO}, \mathrm{H}_{2} \mathrm{O}, \mathrm{OH}$ and ${ }^{13} \mathrm{CO}$, respectively,
$X_{\text {mol }}=(1,0.1,0.02, \geq 0.025) \times 10^{-4}$. The ortho-topara ratio for $\mathrm{H}_{2} \mathrm{O}$ is consistent with the high temperature equilibrium value (ratio of the statistical weights of the nuclear spins), i.e. $\mathrm{H}_{2} \mathrm{O}-o / p=3$.

- The relatively high $\mathrm{OH}$ abundance is indicative of an elevated level of ionising flux in the Serpens cloud core, causing the ionisation rate to be $\zeta \gg 10^{-18} \mathrm{~s}^{-1}$, i.e. significantly higher than the average rate prevailing in dark clouds. Strong and active X-ray sources, known to exist in the cloud, could be responsible for this radiation.

- The observed SED of SMM 1 is consistent with a model of a dusty torus with an outer radius of $14000 \mathrm{AU}$ $\left(45^{\prime \prime}\right)$. The torus is heated by a central stellar source, with a (somewhat arbitrarily) adopted effective temperature of $5000 \mathrm{~K}$. A luminosity of $140 L_{\odot}$ is required to explain the observations. The total mass of the toroidal core of SMM 1 is $33 M_{\odot}$. The derived visual extinction through the torus exceeds 2000 mag and the torus is optically thick up to mm wavelengths.

- 2D modelling of the radiative transfer through the circumstellar torus of SMM 1 reveals that it is highly unlikely that the observed molecular emission arises in the torus. The same conclusion can be drawn for models of dynamical collapse ("inside-out" infall).

- The $\mathrm{H}_{2}$ data have been obtained at significantly higher spatial resolution than that offered by the LWS. These CAM-CVF observations trace the regions of pure rotational $\mathrm{H}_{2}$ line emission. The $\mathrm{H}_{2}$-maxima are observed to be displaced from SMM 1 and are situated toward the northwest, along the jet of outflowing material from this Class 0 source. The temperature of $\sim 10^{3} \mathrm{~K}$ of this $\mathrm{H}_{2}$ gas is indicative of the heating by relatively slow shock waves.

- The comparison of our molecular line data with shock models suggests that shock heating with $v_{\mathrm{s}} \sim(15-20) \mathrm{kms}^{-1}$ along the outflow of SMM 1 is the most likely mechanism of molecular excitation, although the details of these shocks are less clear.

Acknowledgements. We are grateful for the help with the data reductions of the CVF observations by Stephan Ott. We also thank Ewine van Dishoeck for making avalailable to us the collision rate coefficients for $\mathrm{OH}$ in electronic form. The support of this work by Rymdstyrelsen (Swedish National Space Board) is acknowledged.

\section{Appendix A: Reduction of the LWS data}

The photometric calibration of the LWS is primarily based on observations and models of the planet Uranus. The $\mathrm{S} / \mathrm{N}$ in these observations is rather modest, which will affect the relative spectral response function (RSRF) derived from these data. When calibrating the "science data", the registered photocurrent is divided by the RSRF, propagating any uncertainty in the RSRF, which will ultimately lead to errors in the derived flux density, $F_{\lambda}$.

The derived photocurrent after standard processing, resulting in an SPD file and where identified 

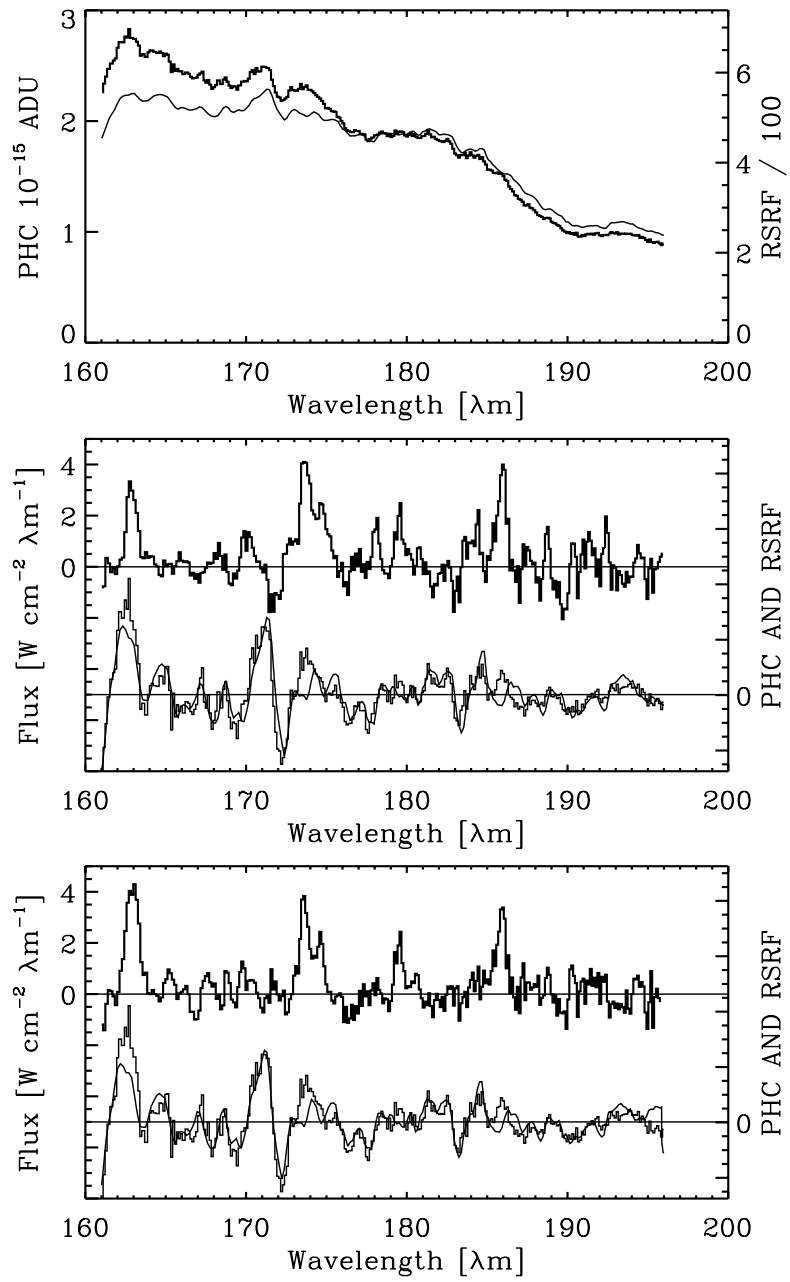

Fig. A.1. Upper panel: the observed wavelength dependence of the photocurrent (thick line) for the detector LW 5 of the LWS. The thin line refers to the grating response function (RSRF) of the detector, scaled to the same mean value as the photocurrent. Middle panel: the lower curves are the same as in the upper panel, after subtracting boxcar fits. The division of the photocurrent by the RSRF results in the upper curve, which is the observed flux density (for clarity a median fit to the continuum has been subtracted). Lower panel: the same as in the middle panel, but with a small wavelength shift $(<0.15 \mu \mathrm{m})$ of the observed photocurrent, before division. Evidently, the "noise" is much reduced: compare, e.g., the spurious "absorption" feature near $172 \mu \mathrm{m}$ in the middle and lower panels.

instrumental peculiarities have been removed, is shown for the LW 5 LWS detector in the upper panel of Fig. A.1, together with the relative spectral response function scaled to the same mean value. From the figure, it is evident that many features seen in the photocurrent are due to the detector and grating response.

The middle panel of Fig. A.1 shows again the photocurrent and the RSRF, but now after the subtraction of a wide boxcar smoothing function (continuum subtraction). The upper curve shows the result of the division of the photocurrent by the response function. This allows us to gauge the effects on the spectra from the narrow features of the RSRF. In the long wavelength regime of the LWS, and in LW 5 in particular, there are spurious absorption features at positions, where the RSRF is steep. In regions, where the RSRF has steep gradients, can already very small wavelength errors create large features that could be mistaken for spectral lines. A wavelength mismatch between the photocurrent and the RSRF will also introduce an overall lowered $\mathrm{S} / \mathrm{N}$.

In the lower panel of Fig. A.1, a relative shift by less than a quarter of an resolution element (the original sampling rate was at four times the spectral resolution) of the photocurrent resulted in the considerable reduction of an apparent broad "absorption" feature near $172 \mu \mathrm{m}$, an overall better $\mathrm{S} / \mathrm{N}$ and therefore a better definition of the spectral lines.

All individual spectra for all ten detectors have been carefully monitored for obviously anomalous features which (most likely) were introduced by the RSRFs. This was done for the RSRFs of both OLP 8 and OLP 10 and any such spurious features were, of course, corrected for. This does not guarantee, however, that no such false spectral features do still exist in our data, as we were very restrictive in our application of any wavelength shifts.

\section{Appendix B: Collision rates for CO}

We used rate coefficents for collisions of $\mathrm{CO}$ with $\mathrm{H}_{2}$ which are based on values found in the literature but which have been extended to rotational quantum numbers $J_{\mathrm{u}}=40$, although extrapolations to higher $J$ is not excluded.

For $J_{\mathrm{u}} \leq 29$ and for low temperatures, $(5-400) \mathrm{K}$, we used the recent rates of Flower (2001; ortho- $\mathrm{H}_{2}-\mathrm{CO}$ and para- $\mathrm{H}_{2}-\mathrm{CO}$; downward rates). For the higher temperature range of $(>400-2000) \mathrm{K}$, the calculations by Schinke et al. (1985; para- $\mathrm{H}_{2}$-CO; upward rates) were used. The matching between these data sets is roughly acceptable, but there exist disagreements (Fig. B.1), which reflect the differences in assumptions and computational methods (see the discussion of resonances by Flower).

In order to arrive at a consistent set of collision rate constants for the hole range of temperatures, the Schinke et al. data (correctly transformed to de-excitation rates; see also: Viscuso \& Chernoff 1988) were laterally shifted to fit the Flower data at $400 \mathrm{~K}$. A satisfactory matching was, however, not really possible for the lowest transitions connecting to the ground state, see the lower panel of Fig. B.1. In the figure, these expanded rates are labelled $\gamma^{\mathrm{I}}$.

These $\gamma^{\mathrm{I}}$ data span $J$-values up to 20 and temperatures between 400 and $2000 \mathrm{~K}$. For the same temperature intervall, McKee et al. (1982, MSWG) have published calculations (He-CO; downward rates) for $J$-values up to 32 (Fig. B.2 upper panel). These rates $(\times 1.37)$ were divided into the Schinke et al. rates and fit by a polynomial to correct the shape of the McKee et al. data (Fig. B.3 upper panel), viz.

$\ln \gamma^{\mathrm{I}} / \ln \gamma_{\mathrm{MSWG}}=a+b J \quad 20 \leq J \leq 32$ 

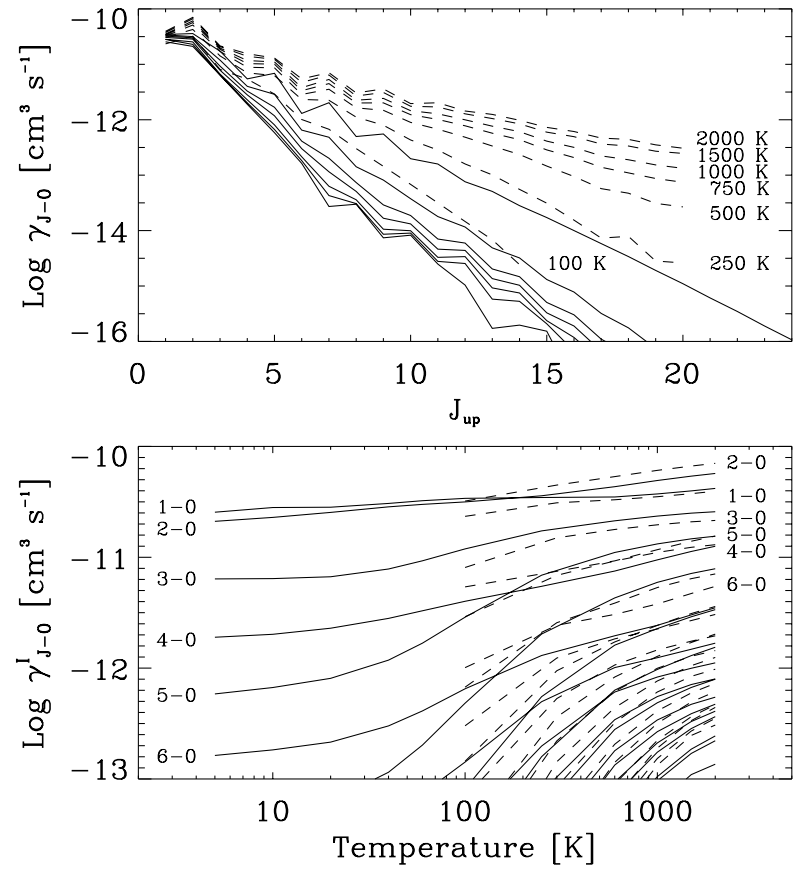

Fig. B.1. Downward rate constants for collisions of $\mathrm{CO}$ with para- $\mathrm{H}_{2}$ : Upper panel: as a function of $J$, where the solid lines are for $T=5,10,20,40,60,100$ and $250 \mathrm{~K}$ from Flower (2001), and the dashed lines for $T=$ $100,250,500,750,1000,1500$ and $2000 \mathrm{~K}$ from Schinke et al. (1985). Lower panel: as a function of $T_{\text {kin }}$, with the data by Schinke et al. adjusted to fit those by Flower at $400 \mathrm{~K}$, expanding the rates for the low temperatures to $T=2000 \mathrm{~K}$ (see the text). These rates are labelled $\gamma^{\mathrm{I}}$ in the figure.

to obtain a new set of rate coefficients

$\gamma^{\mathrm{II}}=\gamma_{\mathrm{MSWG}}^{a+b J}$.

For the expansion to $J=40$, the $\gamma^{\mathrm{II}}$ were fit to a second order polynomial, viz.

$$
\ln \gamma^{\mathrm{II}}=c+d J+e J^{2} \quad 7 \leq J \leq_{32}^{29}
$$

the result of which was used for the extrapolations of the para- $\mathrm{H}_{2}$ rates up to $J=40$, viz.

$$
\gamma^{\mathrm{III}}=\exp \left(c+d J+e J^{2}\right) \quad J>_{32}^{29} .
$$

This provides us finally with the full set of collision rate coefficents for $J_{\mathrm{u}} \rightarrow 0$ for $J_{\mathrm{u}}$ up to 40 (Fig. B.3).

So far, we have considered rates only connecting to the ground state, i.e. $\gamma_{J-0}$. Flower (2001) did provide rates for collision transitions between all level's, but for higher $J$-values and/or higher temperatures we don't have that information. If the kinetic energy of the collision partners on the other hand is large compared to the rotational energy spacing of the $\mathrm{CO}$ molecule, the other rate cofficients can be obtained from (Goldflam et al. 1977; McKee et al. 1982)

$$
\begin{aligned}
\gamma_{J-J^{\prime}}= & \left(2 J^{\prime}+1\right) \times \\
& \times \sum_{L}(2 L+1)\left(\begin{array}{ccc}
J & L & J^{\prime} \\
0 & 0 & 0
\end{array}\right)^{2} A_{L, J}^{2} \gamma_{L-0}
\end{aligned}
$$
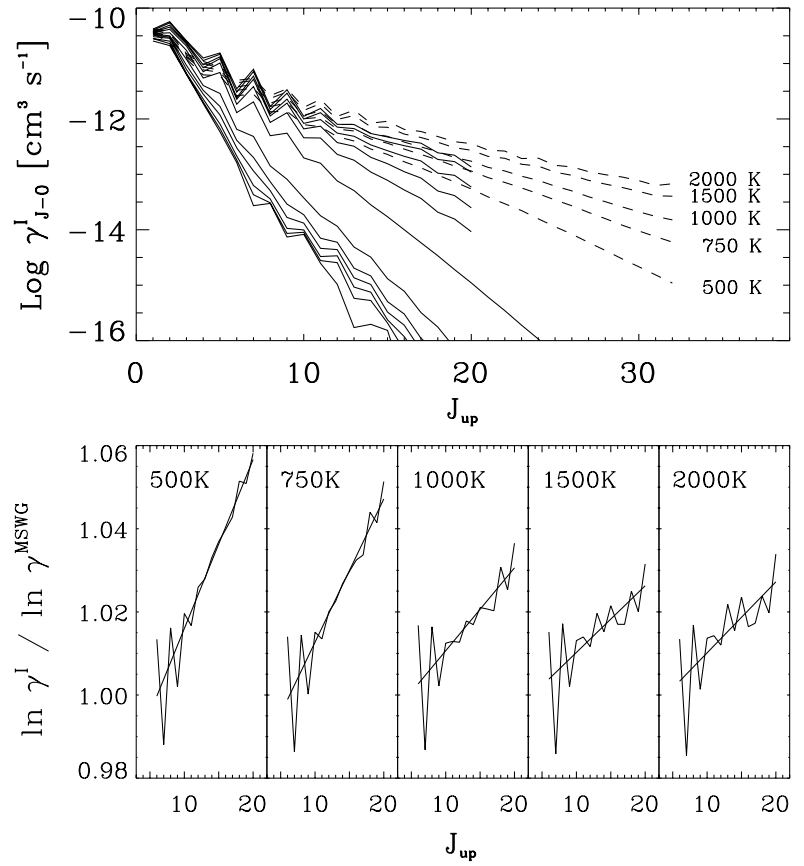

Fig. B.2. Rate constants for $\mathrm{CO}$ as a function of $J$ : Upper panel: the solid lines refer to the rescaled data of Fig. B.1, i.e. $\gamma^{\mathrm{I}}$, whereas the dashes are for $T=$ $500,750,1000,1500$ and $2000 \mathrm{~K}$ from McKee et al. (1982) (collisions with $\mathrm{He}$, scaled to $\mathrm{H}_{2}$, and up to $J=32$ ). Lower panel: the fitted ratios of these rates $\left(J_{\mathrm{u}} \leq 20\right)$ as a function of $J_{\mathrm{u}}$ and, as an example, for the five selected temperatures.

where the Wigner 3-j symbol designates the ClebschGordan coefficients, which were computed exactly (see, e.g., Zare 1986). Further, the factor $A_{L, J}$

$A_{L, J}=\frac{6+(a L)^{2}}{6+(a J)^{2}}$, where $a=0.13 B_{0} l_{\text {sca }}\left(\frac{\mu}{T}\right)^{\frac{1}{2}}$

given by McKee et al. (1982), should correct for the decreased accuracy when the energy splittings become larger. $B_{0}=1.9225 \mathrm{~cm}^{-1}$ is the rotational constant (Lovas et al. 1979), $l_{\mathrm{sca}}=3 \AA$ is a typical scattering length, $\mu=3.5 \mathrm{amu}$ is the reduced mass of the colliding $\mathrm{CO}$ and He particles and $T$ is the kinetic gas temperature.

Finally, the rates for the inverse transitions were obtained from the condition of detailed balancing, viz.

$\left(2 J^{\prime}+1\right) \gamma_{J^{\prime}-J}=(2 J+1) \gamma_{J-J^{\prime}} \exp \left(-h \nu_{J-J^{\prime}} / k T\right)$.

For ${ }^{13} \mathrm{CO}$, we used the same rate constants as for $\mathrm{CO}$, with the proviso that the mass difference potentially introduces an additional error on the collision rates (3.5\%).

\section{References}

Abgrall, H., \& Roueff, E. 1989, A\&AS, 79, 313

André, P., Ward-Thompson, D., \& Barsony, M. 1993, ApJ, 406, 122

Bragg, S. L., Brault, J. W., \& Smith, W. H. 1982, ApJ, 263, 999

Brown, D. W., Chandler, C. J., Carlstrom, J. E., et al. 2000, MNRAS, 319, 154 

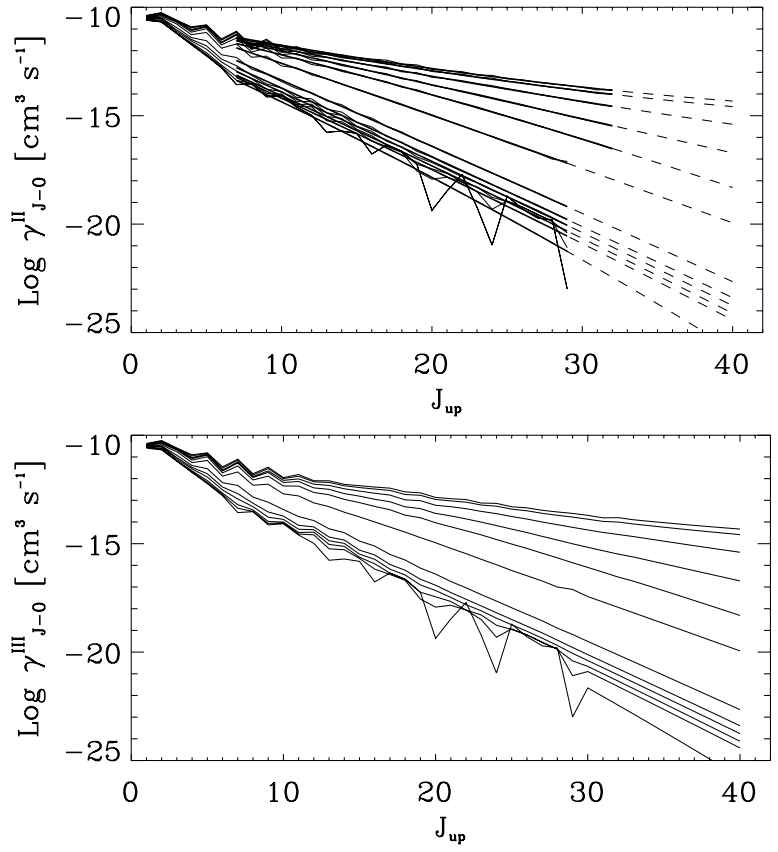

Fig. B.3. Upper panels: applying the curvature corrections to the data by McKee et al. results in the rates named $\gamma^{\mathrm{II}}$ and shown by the solid lines. The extrapolations of $\gamma^{\mathrm{II}}$ to high $J$-values are indicated by the dashed lines. Lower panel: the finally adopted collision rate coefficients for $\mathrm{CO}, \gamma^{\mathrm{III}}$, for the hole range in temperature, $T=(5-2000) \mathrm{K}$, and energy, $J=0-40$.

Cameron, M., \& Liseau, R. 1990, A\&A, 240, 409

Casali, M. M., Eiroa, C., \& Duncan, W. D. 1993, A\&A, 275, 195

Castor, J. I. 1970, MNRAS, 149, 111

Cesarsky, C. J., Abergel, A., Agnèse, P., et al. 1996, A\&A, 315, L32

Chandra, S., Varshalovich, D. A., \& Kegel, W. H. 1984, A\&AS, 55,51

Chandra, S., Maheshwari, V. U., \& Sharma, A. K. 1996, A\&AS, 117,557

Clegg, P. E., Ade, P. A. R., Armand, C., et al. 1996, A\&A, 315, L38

Dame, T. M., Ungerechts, H., Cohen, R. S., et al. 1987, ApJ, 322,706

Davis, C. J., Matthews, H. E., Ray, T. P., Dent, W. R. F., \& Richer, J. S. 1999, MNRAS, 309, 141

de Lara, E., Chavarria, K. C., \& López-Molina, G. 1991, A\&A, 243, 139

Eiroa, C., \& Casali, M. M. 1989, A\&A, 223, L17

Eiroa, C., Torrelles, J. M., Gómez, J. F., et al. 1992, PASJ, 44, 155

Flower, D. R. 2001, J. Phys. B: At. Mol. Opt. Phys., 34, 1

Goldflam, R., Green, S., \& Kouri, D. J. 1977, J. Chem. Phys., 67,4149

Goldsmith, P. F., \& Langer, W. D. 1999, ApJ, 517, 209

Green, S., Maluendes, S., \& McLean, A. D. 1993, ApJS, 85, 181

Gregersen, E. M., Evans II, N. J., Zhou, S., \& Choi, M. 1997, ApJ, 484, 256

Harvey, P. M., Wilking, B. A., \& Joy, M. 1984, ApJ, 278, 156

Hodapp, K. W. 1999, AJ, 118, 1338

Hogerheijde, M. R., van Dishoeck, E. F., Salverda, J. M., \& Blake, G. A. 1999, ApJ, 513, 350
Hollenbach, D. J. 1985, Icarus, 61, 36

Hollenbach, D. J., Chernoff, D. F., \& McKee, C. F. 1989, ESA SP-290, 245

Hollenbach, D., \& McKee, C. F. 1989, ApJ, 342, 306

Kaas, A. A. 1999, Ph.D. Thesis, Stockholm University

Kaufman, M. J., \& Neufeld, D. A. 1996, ApJ, 456, 611

Kessler, M. F., Steinz, J. A., Anderegg, M. E., et al. 1996, A\&A, 315, L27

Lada, C. J., \& Wilking, B. A. 1984, ApJ, 287, 610

Larsson, B., Liseau, R., Men'shchikov, A. B., et al. 2000, A\&A, 363,253

Leung, C.-M., \& Liszt, H. S. 1976, ApJ, 208, 732

Liseau, R., Huldtgren, M., Fridlund, C. V. M., \& Cameron, M. 1996a, A\&A, 306, 255

Liseau, R., Ceccarelli, C., Larsson, B., et al. 1996b, A\&A, 315, L181

Liseau, R., Giannini, T., Nisini, B., et al. 1997, in Herbig-Haro Flows and the Birth of Low Mass Stars, ed. B. Reipurth, \& C. Bertout, 111

Liseau, R., White, G. J., Larsson, B., et al. 1999, A\&A, 344, 342

Lovas, F. J., Johnson, D. R., \& Snyder, L. E. 1979, ApJS, 41, 451

Mardones, D., Myers, P. C., Tafalla, M., et al. 1997, ApJ, 489, 719

McKee, C. F., Storey, J. W. V., \& Watson, D. M. 1982, ApJ, 259,647

McMullin, J. P., Mundy, L. G., Wilking, B. A., et al. 1994, ApJ, 424, 222

McMullin, J. P., Mundy, L. G., Blake, G. A., et al. 2000, ApJ, 536,845

Men'shchikov, A. B., \& Henning, Th. 1997, A\&A, 318, 879

Men'shchikov, A. B., Henning, Th., \& Fischer, O. 1999, ApJ, 519,257

Neufeld, D. A., \& Hollenbach, D. J. 1994, ApJ, 428, 170

Nordh, L., van Duinen, R. J., Sargent, A. I., et al. 1982, A\&A, 115,308

Offer, A. R., van Hemert, M. C., \& van Dishoeck, E. F. 1994, J. Chem. Phys., 100, 362

Ossenkopf, V., \& Henning, Th. 1994, A\&A, 291, 943

Palla, F., \& Stahler, S. W. 1990, ApJ, 360, L47

Rodríguez, L. F., Curiel, S., Moran, J. M., et al. 1989, ApJ, 34, L85

Sandford, S. A., \& Allamandola, L. J. 1993, ApJ, 417, 815

Schinke, R., Engel, V., Buck, U., Meyer, H., \& Diercksen, G. H. F. 1985, ApJ, 299, 939

Sharpless, S. 1959, ApJS, 4, 257

Shu, F. H., Adams, F. C., \& Lizano, S. 1987, ARA\&A, 25, 23

Smith, K., Güdel, M., \& Benz, A. O. 1999, A\&A, 349, 475

Strom, S. E., Vrba, F. J., \& Strom, K. M. 1976, AJ, 81, 638

Swinyard, B. M., Clegg, P. E., Ade, P. A. R., et al. 1996, A\&A, 315, L43

Testi, L., Sargent, A. I., Olmi, L., \& Onello, J. S. 2000, ApJ, 540, L53

Viscuso, P. J, \& Chernoff, D. F. 1988, ApJ, 327, 364

Wardle, M. 1999, ApJ, 525, L101

White, G. J., Casali, M. M., \& Eiroa, C. 1995, A\&A, 298, 594

White, G. J., Liseau, R., Men'shchikov, A. B., et al. 2000, A\&A, 364, 741

Williams, J. P., \& Myers, P. C. 2000, ApJ, 537, 891

Winkler, K.-H.A., \& Newman, M. J. 1980, ApJ, 236, 201

Wolniewicz, L., Simbotin, I., \& Dalgarno, A. 1998, ApJS, 115, 293

Zare, R. N. 1986, Angular Momentum (J. Wiley \& Sons)

Ziener, R., \& Eislöffel, J. 1999, A\&A, 347, 565 